\title{
The Healthy Female Microbiome Across Body Sites: Effect of Hormonal Contraceptives and the Menstrual Cycle
}

\section{Maria Christine Krog}

Copenhagen University

\section{Luisa W Hugerth}

Kl: Karolinska Institutet https://orcid.org/0000-0001-5432-1764

\section{Emma Fransson}

$\mathrm{Kl}$ : Karolinska Institutet

\section{Zahra Bashir}

Slagelse Hospital: Slagelse Sygehus

\section{Anders Nyboe Andersen}

Rigshospitalet

\section{Gabriella Edfeldt}

KI: Karolinska Institutet

\section{Lars Engstrand}

Kl: Karolinska Institutet

Ina Schuppe-Koistinen ( $\boldsymbol{D}$ ina.schuppe.koistinen@ki.se)

Kl: Karolinska Institutet

Henriette Svarre Nielsen

Copenhagen University; Hvidovre Hospital

\section{Research}

Keywords: Hormonal contraceptives, Microbiome, Womens reproductive health, Shotgun sequencing Posted Date: August 30th, 2021

DOI: https://doi.org/10.21203/rs.3.rs-696563/v2

License: (c) (i) This work is licensed under a Creative Commons Attribution 4.0 International License. Read Full License 


\section{The healthy female microbiome across body sites: Effect of hormonal contraceptives and the menstrual cycle}

Maria Christine Krog*1,2 and Luisa W. Hugerth*3,4, Emma Fransson ${ }^{3,4}$, Zahra Bashir $^{1,5}$, Anders Nyboe Andersen ${ }^{6}$, Gabriella Edfeldtt, ${ }^{3,4}$. Lars Engstrand ${ }^{3,4}$, , Henriette Svarre Nielsen ${ }^{* 1,2,7}$ Ina Schuppe-Koistinen ${ }^{\sharp * 3,4}$

${ }^{1}$ The Recurrent Pregnancy Loss Unit, The Fertility Clinic, The Capital Region, Rigshospitalet and Hvidovre Hospital, Copenhagen University Hospitals, Denmark

${ }^{2}$ Department of Clinical Medicine, Copenhagen University, Copenhagen, Denmark

${ }^{3}$ Centre for Translational Microbiome Research, Department of Microbiology, Tumor and Cell Biology (MTC), Karolinska Institutet, Stockholm, Sweden ${ }^{4}$ Science for Life Laboratory, Stockholm, Sweden

${ }^{5}$ Department of Obstetrics and Gynecology, Slagelse Hospital, Slagelse, Denmark ${ }^{6}$ The Fertility Clinic, section 4071, Rigshospitalet, Copenhagen University Hospital, Denmark

${ }^{7}$ Department of Gynecology and Obstetrics, Hvidovre Hospital, Copenhagen, Denmark

*Shared authorship

${ }^{¥}$ Corresponding author: Ina Schuppe Koistinen: ina.schuppe.koistinen@ki.se 


\section{Abstract}

Background: There is increasing evidence that the vaginal microbiome influences women's health. Vaginal dysbiosis with a low abundance of Lactobacillus has been connected to gynaecological and obstetric complications. However, the interplay between the vaginal microbiome and the microbiome in other body sites is not yet described. In addition, fluctuating endogenous sex hormones may exert an effect on microbiome composition and are markedly changed by hormonal contraception. This study includes a cohort of 160 healthy young Caucasian women using three different contraceptive regimens: non-hormonal methods, combined oral contraceptive (COC) or levonorgestrel intra-uterine system (LNG-IUS). The oral, vaginal, rectal and faecal microbiomes are characterized by shotgun sequencing during each phase of the menstrual cycle (menses, follicular and luteal phases). Results: The use of COC and LNG-IUS do not affect the microbiome composition or diversity. However, an increased diversity in the vaginal microbiome is observed during menses, followed by a subsequent expansion of Lactobacillus during the follicular and luteal phases which correlates with measured serum oestradiol levels $(r=0.11, p<0.001)$. During menses 89 women (58\%) have a dysbiotic vaginal microbiome with less than 60\% Lactobacillus spp. This declines to $49(32 \%)$ in the follicular phase $(p=1.3 \mathrm{E}-5)$ and $44(29 \%)$ in the luteal phase $(\mathrm{p}=4.6 \mathrm{E}-7)$. During menses, bacterial richness and diversity in saliva reach its lowest while no difference is observed in the faecal microbiome. The microbiome in different body sites is on average more similar within the same individual than between individuals, despite phase or hormonal treatment. Only the vagina presents a clear cluster structure with dominance of either Lactobacillus crispatus, Lactobacillus iners, Gardnerella vaginalis or Prevotella spp.

Conclusions: The use of COC or LNG-IUS is not associated with changes of the healthy female microbiome except for increased stability in the vagina in LNG-IUS users. Body site is the main driver of the microbiome including a clear difference between faecal and rectal samples. The menstrual cycle is a confounding factor for microbiome composition in both saliva and the vagina and should be considered when analysing the microbiome in women of reproductive age.

\section{Keywords (3-10)}

Hormonal contraceptives, Microbiome, Womens reproductive health, Shotgun sequencing 


\section{Introduction}

Microbial communities inhabit every inch of the human body and are thought to actively contribute to the homeostasis and health of every individual [1]. The implications of the microbiota in various body sites and their potential interaction and individual importance in maintaining general health are, however, inadequately understood. Most studies focus on one body site at a time and extensive work have been published documenting associations between the digestive tract microbiome and numerous health conditions, from gastrointestinal diseases to mental illnesses [2]-[6]. The most studied niche in the female reproductive tract is the vaginal microbiome which has been connected to gynaecological health [7], [8].

The gut and oral microbiomes have been shown to have systemic effects and the question remains whether there is an interaction between the microbiota in various body sites and how they could potentially impact women's reproductive health.

Among the most common colonizers of the vaginal tract in reproductive aged women are Lactobacillus species which have been established as the healthy vaginal microbiome linked to positive health outcomes [8]-[13]. Some women of reproductive age lack this Lactobacillus dominance in the vagina but have a diverse composition of other bacteria including anaerobic bacteria. This is similarl to the microbial composition in bacterial vaginosis, a condition characterized by thin, greyish vaginal discharge, unpleasant odour and increased vaginal $\mathrm{pH}$. While common, especially among women of African descent[14]-[16], this microbial composition has been connected to susceptibility to sexually transmitted infections[17], [18], difficulties achieving pregnancy after fertility treatments[10], [19], pre-term birth[20], [21], HPV infection and gynaecological cancers[12], [22]. This has led many research groups to classify a diverse vaginal microbiome with low abundance of Lactobacillus spp. as dysbiotic, even in the absence of symptoms [23], [24]. Interestingly, it is suspected that Lactobacillus dominance in the vagina is estradiol- and/or progesterone-dependent as it is more common in the reproductive years of life, leaving girls and postmenopausal women with a more diverse microbiome with less abundance of Lactobacillus [25]-[27].

The possible effect of hormonal contraception on the vaginal microbiome is debated. Some studies argue for a beneficial impact of the synthetic oestrogens in the combined oral contraceptive pill (COC) favouring Lactobacillus dominance[28], [29] others find no impact on the vaginal microbiome[30], [31]. A more diverse vaginal microbiome in levonorgestrel intrauterine systems (LNG-IUS) 
users have been reported[32], although this alteration has also been found to be temporarily after insertion[33] . Many, especially younger women, use the monophasic COC and are continually exposed to the synthetic ethinyl oestradiol and a progestin. Both hormones work synergistically to suppress ovulation[34]. Whereas most women using the progestin-only (LNG-IUS) continue to ovulate but with suppression of endometrial growth - resulting in less or absent menstrual bleeding [35]. The possible systemic effect of synthetic hormones on the microbiome composition across the body has not been extensively investigated to date, although we have demonstrated some effects for saliva [36].

Extensive work by Ravel's group has categorized specific clusters of microorganisms in the vagina and described the temporal dynamics of the composition in the individual woman[9], [37]. They found that some women remain stable and others shift between a Lactobacillus dominated microbiome and a more diverse community state type over time [37]. Previous studies of the vaginal microbiome have primarily used 16S rRNA gene sequencing but with time, shotgun metagenomic sequencing techniques have emerged which can provide an extensive mapping of all DNA present in the sample.

No previous studies have investigated the longitudinal effect of the fluctuations of sex hormones during the menstrual cycle and the possible effect of hormonal contraception on the composition of the microbiome across body sites. This study compares the shotgun metagenomic profiles of four different body sites in women during a natural menstrual cycle and in women using COC or LNG-IUS to explore an impact of supressed ovulation, menstrual bleeding and shifts in sex hormones on the overall microbiome composition.

\section{Methods}

\section{Participant recruitment}

Women were recruited by advertisements in student magazines, university noticeboards and social media and 160 women were included between September 2017 and January 2018 at Copenhagen University Hospital, Rigshospitalet, Denmark. Women were excluded if they were pregnant or planning to become pregnant during the study period of 6 weeks or were taking or had taken antimicrobial medication (antibiotics, antiviral or antifungal medication) 14 days prior to enrolment. If participants had to use any kind of antimicrobial medication or vaginal lactic acid suppositories, they completed the project but were replaced one to one and their data are not included in this study. Women were recruited to fit into three contraception groups: Group 1, women with a regular menstrual cycle and no 
use of hormonal contraception (NHC), Group 2, women using combined (ethinyl oestradiol and progestin) monophasic oral contraceptive (COC) and Group 3, women using levonorgestrel intrauterine system (LNG-IUS). Women in each group had a regular cycle for at least six months prior to commencing their contraceptive regimen. The three contraceptive regimens were chosen because they are commonly used in women of reproductive age and also provide a unique opportunity to investigate the effects of the fluctuations in sex hormones (NHC group), suppressed ovulation without progesterone rise in the luteal phase (COC group) and sparse/absent menstrual bleeding (LNG-IUS).

\section{Sample collection}

Women were followed during a six-week period including three hospital visits (fig.1). The participants contacted the study coordinator at the first day of bleeding and was then scheduled for the first hospital visit cycle day (CD) 13. If the participant had oligo-/amenorrhoea (group 3, LNG-IUS, $n=32$ ), she was scheduled at a random day. The second visit was scheduled at CD 8-12 and the third CD 18-22, representing the follicular and luteal phase of the menstrual cycle. For women with oligo-/amenorrhoea, the assignment to cycle phase was performed later, based on serum levels of oestradiol and progesterone.

Vaginal swabs, saliva and blood samples were collected at every hospital visit and a rectal sample was collected at the first hospital visit. After each hospital visit, participants collected a stool sample at home within 48 hours. The faecal samples were collected with Zymo’s DNA/RNA-shield Fecal Collection Tube and stored at ambient temperature until the next hospital visit.

At every hospital visit the women underwent a gynaecological examination by medical staff including a transvaginal ultrasound to exclude pathological conditions in the uterus or a pregnancy. A rectal sample was obtained by inserting the swab approximately $1-2 \mathrm{~cm}$ in the rectum and the swab was turned three times, touching the walls of the rectum. Vaginal and rectal samples were taken with FLOQSwabs (Copan Flock Technologies, Brescia, Italy) and put directly into FluidX tubes (Brooks Life Sciences, Chelmsford, MA, USA) containing $0.8 \mathrm{ml}$ DNA/RNA-shield (Zymo Research, Irvine, CA, USA).

The patients were fasting 30 minutes before saliva collection, including drinking, chewing gum and smoking. Saliva samples were collected in a SalivaGene Collector (STRATEC Molecular GmbH, Germany) containing lyophilized DNA stabilization buffer, according to the instructions of the manufacturer.

Blood samples were collected at the three hospital visits during the menstrual cycle in $9 \mathrm{ml}$ EDTA tubes and plasma was stored in $-80^{\circ}$ degrees. 
Oestradiol and progesterone were measured in plasma using the standard automated system (Cobas ${ }^{\circledR} 8000$ by Roche Diagnostics). Associations between sex hormones during the menstrual cycle and the microbiome were only calculated for women not using hormonal contraception, as women using hormonal contraceptives are influenced by other synthetic sex hormone derivatives (such as ethinyl oestradiol and progestins), not routinely measured in plasma.

Because of the large prevalence of absent menstrual bleeding in women using LNG-IUS ( $\mathrm{n}=32$ ), the order of samples was adjusted based on their progesterone levels, with the sample with the highest progesterone level being set as "luteal" and the others distributed accordingly (fig. S1).

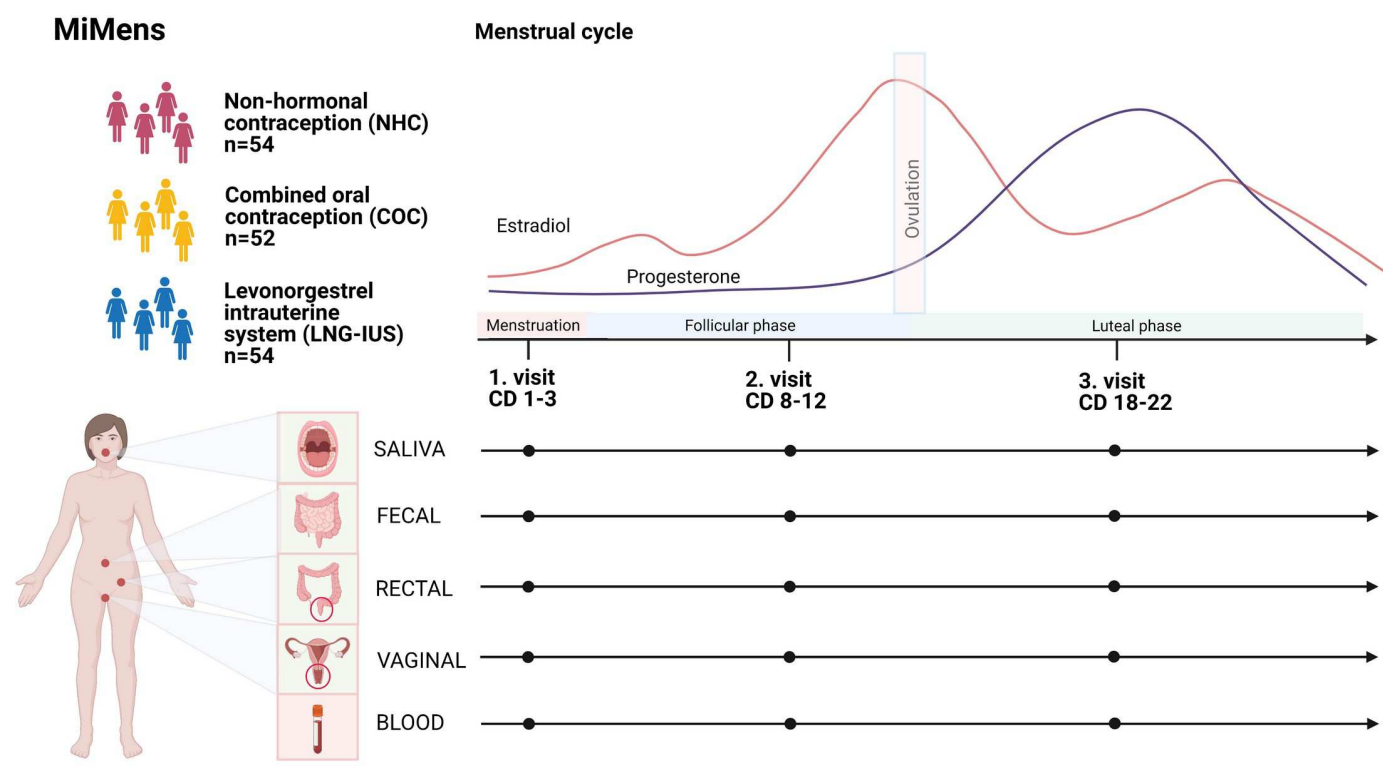

Figure 1: Schematic representation of the sampling scheme

Over 50 women were recruited using one of three contraceptive regimens: NHC (non-hormonal contraceptives), COC (combined oral contraceptives) and LNG-IUS (levonorgestrel intra-uterine system). Included women were sampled at the hospital during the menstrual phase (cycle days 1-3), follicular phase (cycle days 8-12) and luteal phase (cycle days 18-22). Blood samples, saliva and rectal swabs were collected at the hospital; vaginal swabs and faeces were collected by the women at home. Women on LNG-IUS with oligo-/amenorrhoea started their sampling at a random day. 

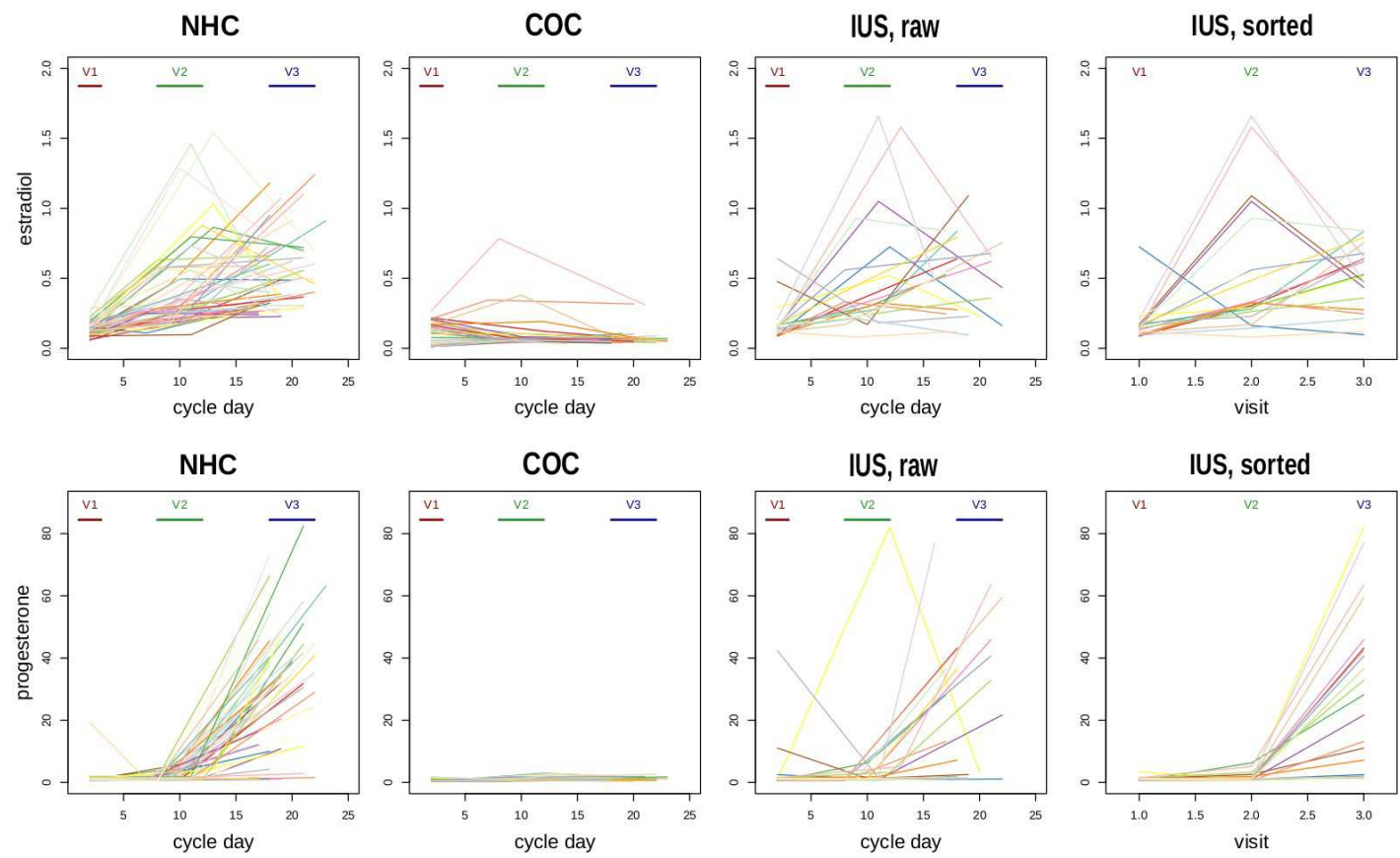

Fig. S1: Oestradiol and progesterone levels for each subject at each sampling time, in $\mathbf{n m o l} / \mathbf{\mu L}$.

Subjects are separated by contraceptive usage. "IUS, raw" shows hormone levels in chronological order, while "IUS, adjusted" shows them reordered so that the progesterone peak is at the third sampling point. This is the order in which IUS samples were analysed throughout the article.

\section{Questionnaires}

At the first hospital visit, the participants answered a comprehensive online questionnaire about general and reproductive health, lifestyle factors such as diet and exercise, and the use of menstrual hygiene products (Questionnaire 1). After every hospital visit the participants received a 48-hour food-recall questionnaire and current gynaecological issues questionnaire (Questionnaire 2). Additionally, they filled out a diary where they registered bleedings (including spotting) and sexual intercourses.

Fibre intake was recorded using a four-week food recall. Participants reported on a 9-point scale from " 0 times in the past four weeks" to " $>3$ times/day for the past four weeks" with the following fibre categories: rye bread; whole wheat bread; fruit; vegetable dishes; salad and prepared vegetables. Intake of at least one daily high fibre category was categorized as “daily”. Free-sugar intake was derived from a similar scale including the items: chocolate milk; juice; soda with sugar; ice- 
cream; biscuits and cookies; sweet bread and rolls; dry cake; cake with filling; candy, as we previously described [36].

Ethics

All participants gave oral and written consent to participate. The participants were remunerated by receiving 3,000 DKK before taxes when they completed participation. All data were collected and managed using REDCap electronic data capture tools [38], hosted at the Capital Region of Denmark. The study is approved by The Regional Committee on Health Research Ethics (H17017580) and the Data Protection Agency in the Capital Region of Denmark (2012-58-0004).

DNA extraction and sequencing

Samples for microbiome analysis were shipped to CoreBiome (OraSure, Bethlehem, PA, USA) where they were extracted with MO Bio PowerFecal kit (Qiagen, Hilden, Germany) automated for high throughput on QiaCube (Qiagen), with bead-beating in $0.1 \mathrm{~mm}$ glass bead plates. Three spaced negative controls and one positive control were included in each extraction. All negative extraction controls had undetectable amounts of DNA, and all positive controls were also approved. The DNA concentration of samples and controls was quantified using Quant-iT Picogreen dsDNA Assay (Invitrogen, ThermoFisher Scientific, Carlsbad, CA, USA).

Libraries were prepared using an adapted Nextera (Illumina Inc, San Diego, CA, USA) procedure and sequenced on an Illumina NextSeq using singleend 150 bp reads with a NextSeq 500/550 High Output v2 kit. Reads were processed with CoreBiome's BoosterShot shallow shotgun sequencing technology.

\section{Annotation of metagenomic reads}

Because the taxonomic annotation of BoosterShot technology is optimized for the human gut, saliva samples were reannotated using Kraken2[39] and Bracken[40] based on the Human Oral Microbiome Database v9.0.3[41]. Similarly, vaginal samples were reannotated with the same tools and the OptiVag database [42]. Due to the uneven amount of annotated bacterial reads generated, each of these samples was filtered to keep species corresponding to at least $0.5 \%$ of annotated reads. Samples not attaining 10000 annotated reads were discarded. Faecal and rectal samples were analysed based on the filtered BoosterShot file. We did not use the annotations described above when comparing strains across body sites, for which raw, unfiltered, BoosterShot annotations were used, as these preserve strain-level information. 


\section{Statistical analyses}

The descriptive table statistics were performed using Kruskal-Wallis and Chi-Square with SPSS (v26.0; IBM Corp, Armonk, NY, USA). All other statistical analyses were performed in R v3.5.2. Alpha-diversity was calculated as the observed number of species as well as Simpson's inverted index. Beta-diversity was calculated on Bray-Curtis distances. Alpha- and beta-diversity were calculated with package Vegan (v2.5-3;[43]) and graphs were generated with packages RColorBrewer (v1.1-2), Vioplot (v0.2) and Pheatmap (v1.0.10). Explorative analysis of possible confounding factors was conducted with a PERMANOVA based on Bray-Curtis distances of each sample type in turn. Significant factors for each body site were treated as a possible confounder for all other body sites. The following factors were considered: Smoking: daily, occasional, not currently; Snus (moist nonsmoke tobacco): current, not-currently, Alcohol: up to 7 weekly units, $>7$ weekly units; Bristol stool form scale: normal transit (3-4), slow transit (1-4), fast (3-7), varied (both 1-2 \& 5-7); BMI: lowest quartile (<=20.6), middle 50\% and top quartile ( $>=23.9$ ); Fiber intake: daily vs non-daily ; Free-sugar consumption: lowest quartile (<=4 occasions/week), middle $50 \%$ and top $25 \%$ ( $>=22.7$ occasions/week). This is described in more detail in Bostanci et al. [36].

Comparisons between alpha-diversity at baseline compared to follicular and luteal phases for the same individual were calculated as paired t-tests, while comparisons across individuals were calculated using Welch's t-test. For binary outcomes (presence/absence), the chi-squared test was used. For betadiversity comparisons, because the distribution for vaginal samples was strongly non-normal, Wilcoxon's rank sum test with continuity correction was used. All tests were performed with a 95\% confidence interval and a significance cut-off of $\mathrm{p}<$ 0.05. Multiple testing correction was conducted with the Benjamini-Hochberg procedure where applicable.

Associations between taxa and hormones or metadata parameters were calculated in Maaslin2 [44]. For the hormonal analysis, serum levels of oestradiol and progesterone were considered as fixed effects, with subject identity as a random effect. For an analysis on the combined effects of contraceptive usage and the menstrual cycle, the following co-variables were included: smoking status, snus usage, alcohol consumption and sugar consumption, with subject identity as a random effect. 


\section{Results}

\section{Cohort description}

Characteristics and health parameters of the participating women in the three contraceptive method groups (no hormonal contraceptives, NHC; combined oral contraceptive, COC and levonorgestrel intrauterine system, LNG-IUS) are presented in table 1. Some differences are observed between the groups, mostly related to reproductive health, with fewer women having a regular menstrual cycle in the LNG-IUS group (57\% vs 100\% in the other groups), but also that fewer women in the COC group report to have a healthy diet (COC: 38\%; NHC: 55\%; LNG-IUS: $68 \%$ ) and a larger proportion of women with LNG-IUS report being in a relationship (78\% vs NHC: $46 \%$ and COC: $50 \%$ ).

\begin{tabular}{|c|c|c|c|c|}
\hline & $\begin{array}{l}\text { No hormonal } \\
\text { contraceptives } \\
\mathrm{N}=54\end{array}$ & 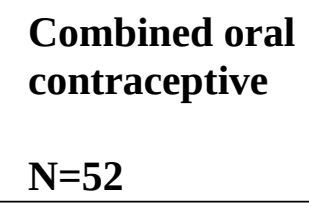 & $N=54$ & p-value \\
\hline \multicolumn{5}{|l|}{ Background } \\
\hline $\begin{array}{l}\text { Born in Denmark (vs } \\
\text { born outside), n \% }\end{array}$ & $47(87.0)$ & $50(96.2)$ & $50(92.6)$ & 0.222 \\
\hline $\begin{array}{l}\text { Age in years, median } \\
\text { (range) }\end{array}$ & 23.5 (19-40) & $23.0(18-36)$ & $23.0(19-40)$ & 0.233 \\
\hline $\begin{array}{l}\text { Level of Education } \\
\text { (ongoing or completed) } \\
\text { University level (vs } \\
\text { less), n (\%) }\end{array}$ & $49(90.7)$ & $49(94.2)$ & $47(87.0)$ & 0.469 \\
\hline $\begin{array}{l}\text { General health } \\
\text { variables }\end{array}$ & & & & \\
\hline $\begin{array}{l}\text { Body mass index (BMI) } \\
\text { Overweight } \mathrm{BMI}>25, \mathrm{n} \\
(\%)\end{array}$ & $12(22.2)$ & 6 (11.5) & $4(7.4)$ & 0.070 \\
\hline $\begin{array}{l}\text { Smoking (at least once } \\
\text { a week), n (\%) }\end{array}$ & $13(24.1)$ & 6 (11.5) & $13(24.1)$ & 0.178 \\
\hline $\begin{array}{l}\text { Snus (at least once a } \\
\text { week), n (\%) }\end{array}$ & $4(7.4)$ & $6(11.5)$ & $4(7.4)$ & 0.687 \\
\hline $\begin{array}{l}\text { Poor self-rated health, } \\
\text { (less than good), n (\%) }\end{array}$ & $5(9.3)$ & $2(3.8)$ & $3(5.6)$ & 0.142 \\
\hline
\end{tabular}




\begin{tabular}{|c|c|c|c|c|}
\hline $\begin{array}{l}\text { Self-rated healthy diet } \\
\text { (vs less healthy), n (\%) }\end{array}$ & $30(55.5)$ & $20(38.4)$ & 37 (68.5) & $<0.001$ \\
\hline $\begin{array}{l}\text { High alcohol } \\
\text { consumption (>7 } \\
\text { units/week), n (\%) }\end{array}$ & $12(22.2 \%)$ & $19(36.5 \%)$ & $13(24.1 \%)$ & 0.202 \\
\hline $\begin{array}{l}\text { Frequent free sugar } \\
\text { consumption (>9 } \\
\text { occasions/week), n (\%) }\end{array}$ & $16(29.6 \%)$ & $16(30.8 \%)$ & $8(14.8 \%)$ & 0.104 \\
\hline Fibers, daily n(\%) & $46(90.2)$ & $44(84.6)$ & $50(92.6)$ & 0.258 \\
\hline \multicolumn{5}{|l|}{ Reproductive health } \\
\hline Have children, n (\%) & $5(9.3)$ & $2(3.8)$ & $6(11.1)$ & 0.365 \\
\hline $\begin{array}{l}\text { Preserved menstrual } \\
\text { cycle, n (\%) }\end{array}$ & $54(100)$ & $52(100)$ & $31(57.4)$ & $<0.001$ \\
\hline $\begin{array}{l}\text { Average days of } \\
\text { bleeding/period, } \\
\text { median (range) }\end{array}$ & $5.5(2.5-8.5)$ & $5(1-10)$ & $2.75(0-12)$ & $<0.001$ \\
\hline $\begin{array}{l}\text { Menstrual cycle length, } \\
\text { median (range) }\end{array}$ & $28(21-39)$ & $27(21-38)$ & $27(22-37)^{a}$ & 0.061 \\
\hline $\begin{array}{l}\text { Symptoms of Bacterial } \\
\text { Vaginosis }^{\mathrm{b}} \\
\text { n (\%) }\end{array}$ & $11(20.4)$ & $10(19.2)$ & $9(16.7)$ & 0.880 \\
\hline In a relationship, $\mathbf{n}(\%)$ & 25 (46.3) & $26(50)$ & $42(77.8)$ & 0.001 \\
\hline Partner & & & & 0.001 \\
\hline Male & $22(40.7)$ & $26(50)$ & $41(75.9)$ & \\
\hline Female & $3(5.5)$ & 0 & $1(1.9)$ & \\
\hline Single & $29(53.7)$ & $26(50)$ & $12(22.2)$ & \\
\hline $\begin{array}{l}\text { Number of sexual } \\
\text { intercourses during the } \\
\text { study period, mean } \\
\text { (SD) }\end{array}$ & $4.4(4.6)$ & $3.4(4.1)$ & $5.2(4.3)$ & 0.049 \\
\hline
\end{tabular}

${ }^{a}$ Only for those with a preserved menstrual cycle

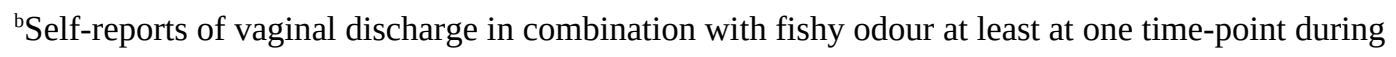
the study period.

Table 1. Background characteristics of participants in the three contraceptive methods groups; non hormonal contraceptives, combined oral contraceptives and levonorgestrel intrauterine system (LNGIUS). 
The following factors correlate by PERMANOVA with the overall spread in microbial samples and are adjusted for in following analyses: sugar consumption, smoking status, snus usage and alcohol intake.

\section{Taxonomic composition across body sites}

Vaginal samples are dominated by Lactobacillus and Prevotella species as well as Gardnerella vaginalis (fig. 2). Saliva samples are dominated by Haemophilus, Neisseria, Prevotella, Streptococcus and Veillonella, regardless of contraceptive method or phase of the menstrual cycle (fig. 3). Faecal samples are dominated by Bacteroides, Allistipes and Faecalibacterium regardless of contraceptive method or phase of the menstrual cycle (fig.3). Rectal samples, which were only collected at the first hospital visit, differ from faecal samples in that they have higher diversity and a larger proportion of unclassified Bacteroidales (fig. 3). No species are associated to hormonal contraceptive usage or the menstrual cycle in saliva, faecal or rectal samples.

The number of strains retained over time in each body site for a given individual is significantly higher than the overlap across individuals $(\mathrm{p}<\mathrm{E}-15$, all sites). There is a significant overlap of shared strains between faecal and rectal samples. Sixty-two genera are found with at least 10 strains in each body site. For these genera, the overlap of strains between body sites is assessed for the first time point (fig. 4). Of interest, the Lactobacillus genus has significantly more strains shared between the rectum and all other body sites. While rectal, faecal and saliva samples all share Lactobacillus strains, the vaginal samples only show significant overlap with rectal samples. Rectal and faecal samples present a large overlap of $L$. paracasei, $L$. casei and $L$. plantarum and rectal samples share strains of $L$. amylovorus with saliva samples and $L$. salivarius, L. acidophilus and $L$. vaginalis with vaginal samples. Saliva samples share $L$. antri, L. fermentum and L. brevis with faecal samples. 

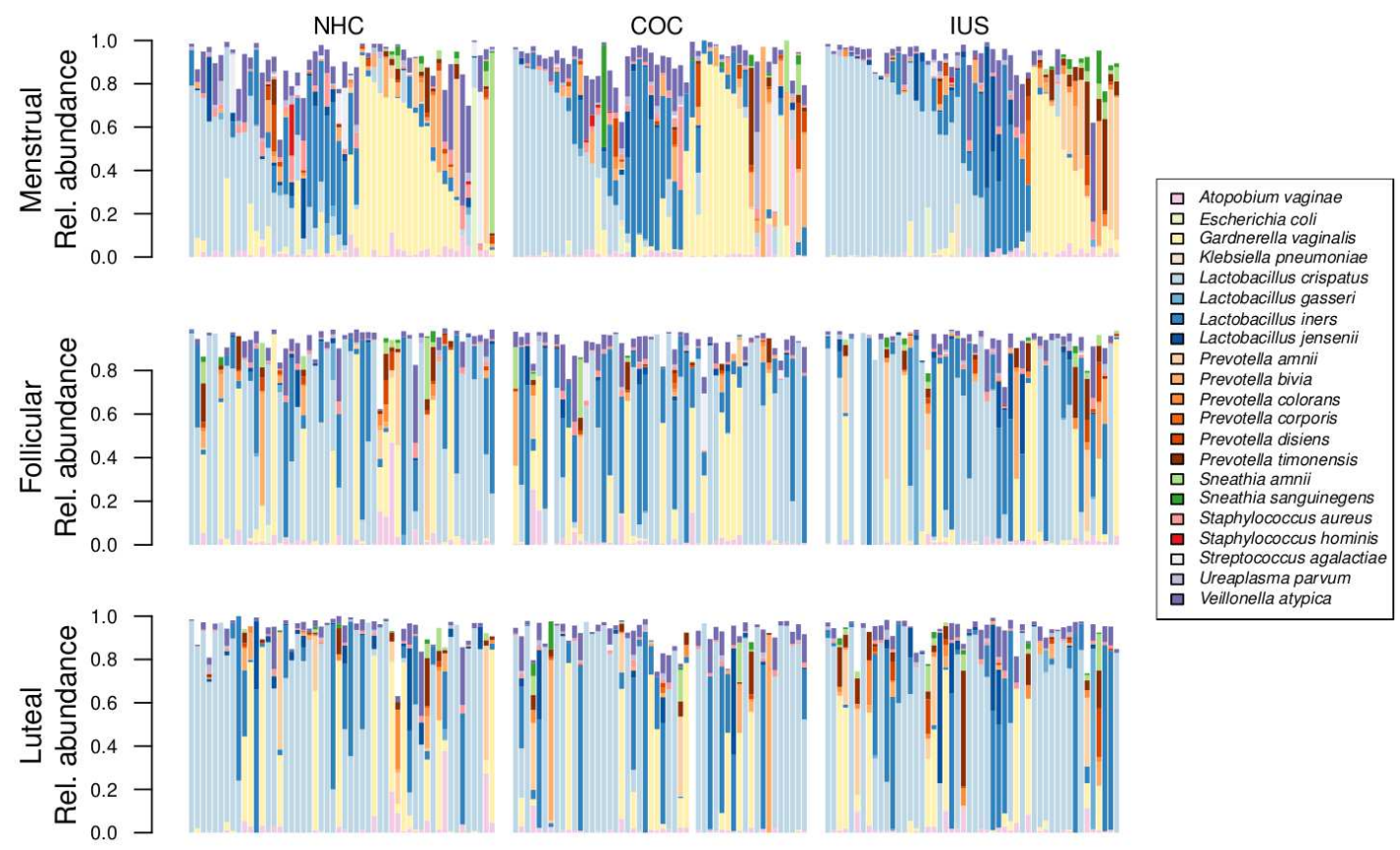

Veillonella atypica

Figure 2: Taxonomic profiles of vaginal samples across contraceptive groups and phase of the menstrual cycle

Vaginal samples are dominated by Lactobacillus crispatus, Lactobacillus iners and Gardnerella vaginalis, as well as various Prevotella spp. Women are separated by contraceptive and sorted after their dominant group in the menstrual phase and are in the same order in each panel, so each individual's progression through the menstrual cycle can be followed directly. Some women's microbiome remains fairly consistent across all three time-points, while others exhibit sharp changes. 

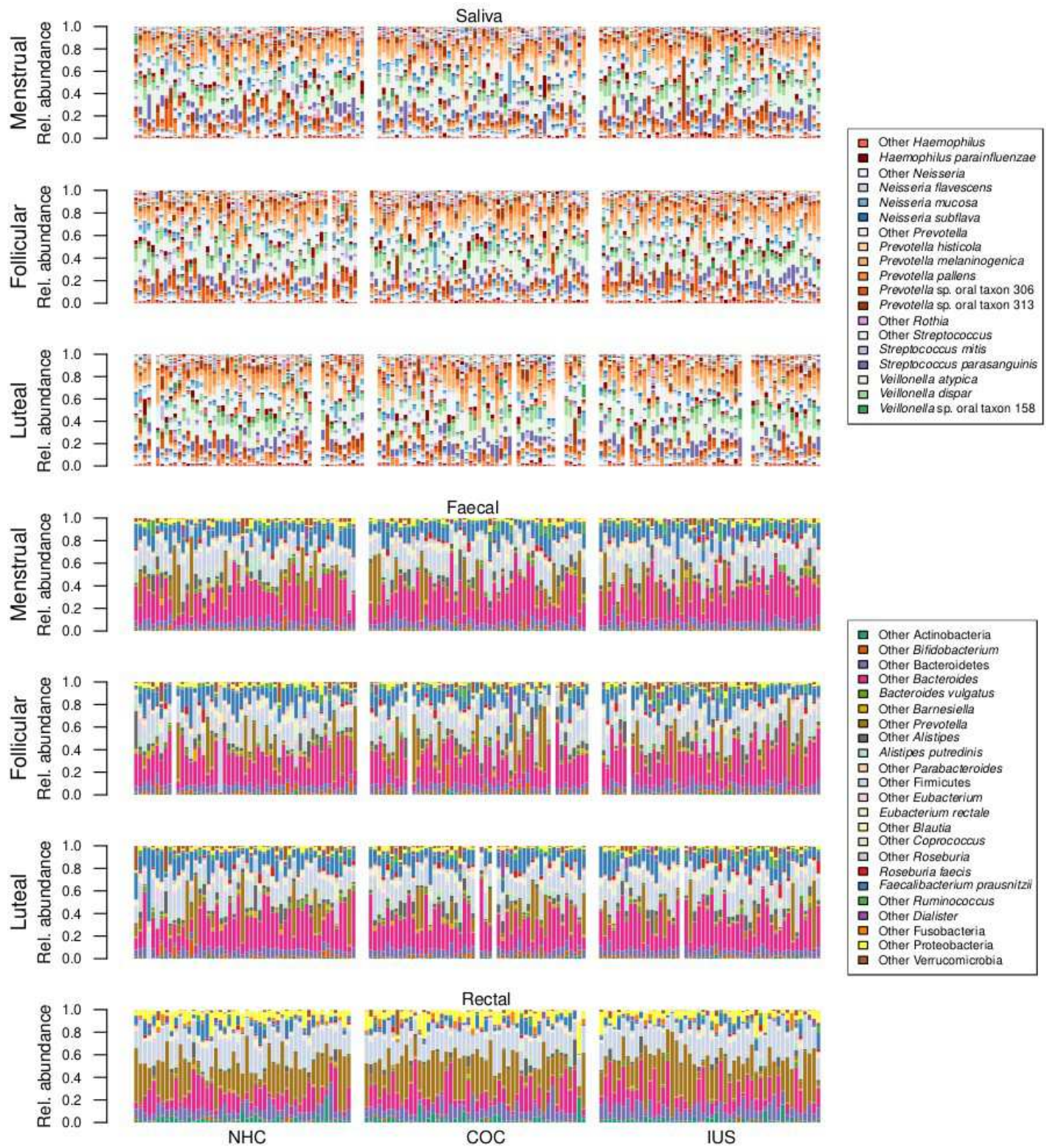

Figure 3: Taxonomic profiles of saliva, faecal and rectal samples across contraceptive groups and phase of the menstrual cycle

Saliva samples are dominated by Veillonella spp, Neisseria spp, Haemophilus spp and Prevotella spp, while faecal and rectal samples are dominated by Faecalibacterium prausnitzii, Bacteroides spp, Roseburia spp and Prevotella spp, the latter being more abundant in the rectum than in faeces. Samples are remarkably stable over time. Women are depicted in the same order as in figure 2, to allow cross-body comparisons as well as temporal follow-up within each body site. 


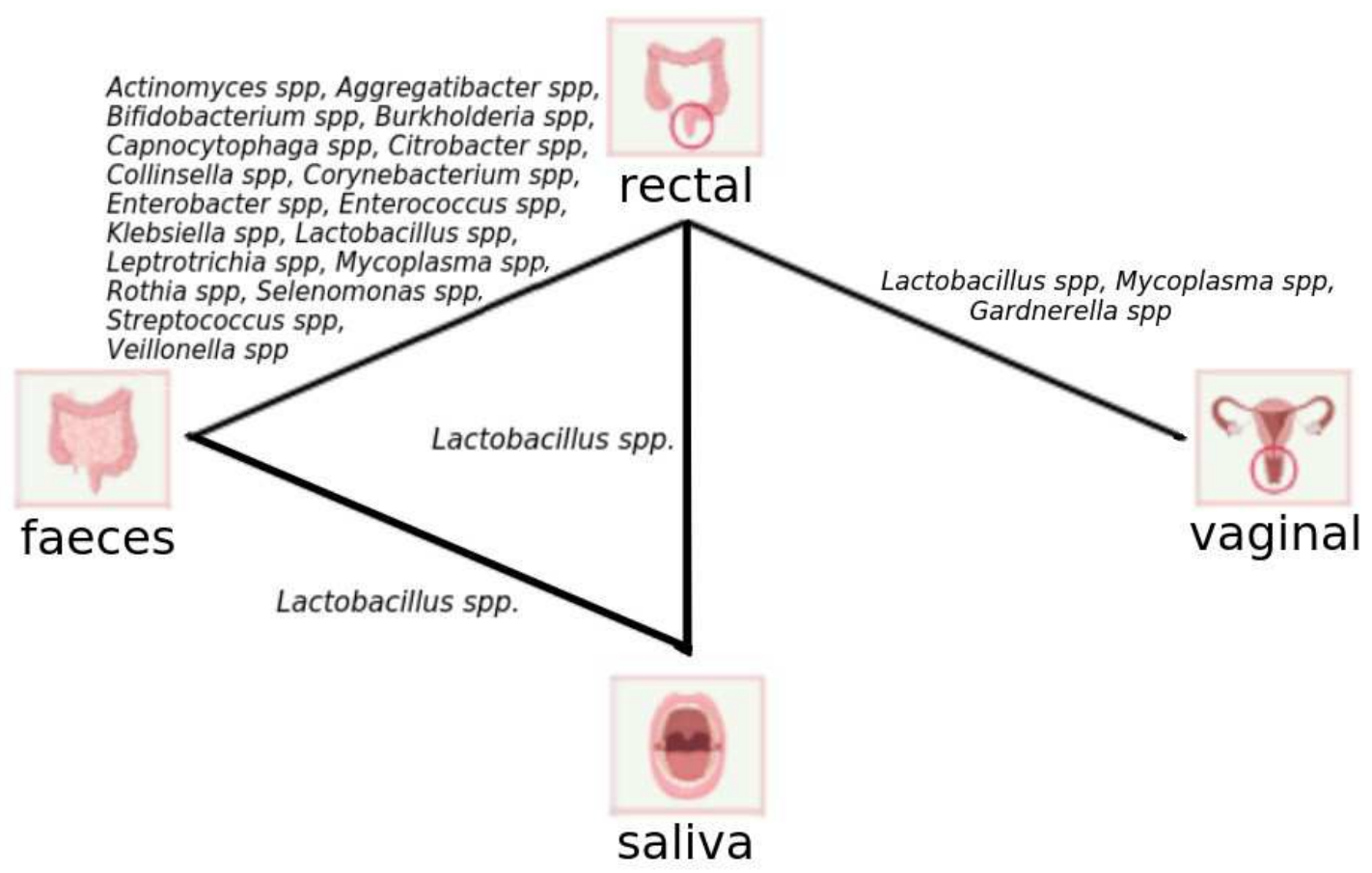

Figure 4: Genera with significant shared strains across body sites

Genera with at least 10 strains in every body site were analysed ( $n=63$ genera). For each genus, we tested whether separate body sites within the same individual shared strains to a larger extent than body sites from different individuals. Genera for which a few strains are prevalent over the majority of individuals can therefore not be shown to be significantly shared in this analysis.

\section{Alpha-diversity and species richness}

No significant differences in alpha-diversity (fig. 5) and species richness (fig. S2) are observed between contraceptive groups. For each individual, rectal samples present the highest richness, followed by faecal, saliva and vaginal samples (paired t-tests, $\mathrm{p}<\mathrm{E}$-4) (fig. 5). Faeces are to a larger extent than saliva dominated by a few species, so despite their higher richness, they have slightly lower diversity (paired ttest, $\mathrm{p}<\mathrm{E}-5$ ). Vaginal samples have significantly higher diversity during the menstrual phase compared with the follicular or luteal phase (both $\mathrm{p}<\mathrm{E}-5$ ). During the menstrual phase, bacterial richness and diversity in saliva reach its lowest, while the vaginal microbiome is at its highest diversity (fig. S2); these differences are only significant for vaginal samples (both $\mathrm{p}<\mathrm{E}-5$ ). Faecal alphadiversity is positively correlated to rectal (Pearson's $r=0.27, p=0.0009$ ) and vaginal diversity $(r=0.11, p=0.02)$, and negatively correlated to oral diversity $(r=-$ $0.12, p=0.01$ ). The other body sites' diversity indexes are not significantly correlated. The negative correlation between oral and faecal diversity is also supported by an inverse correlation in their richness $(r=-0.16, p=0.0007)$. 


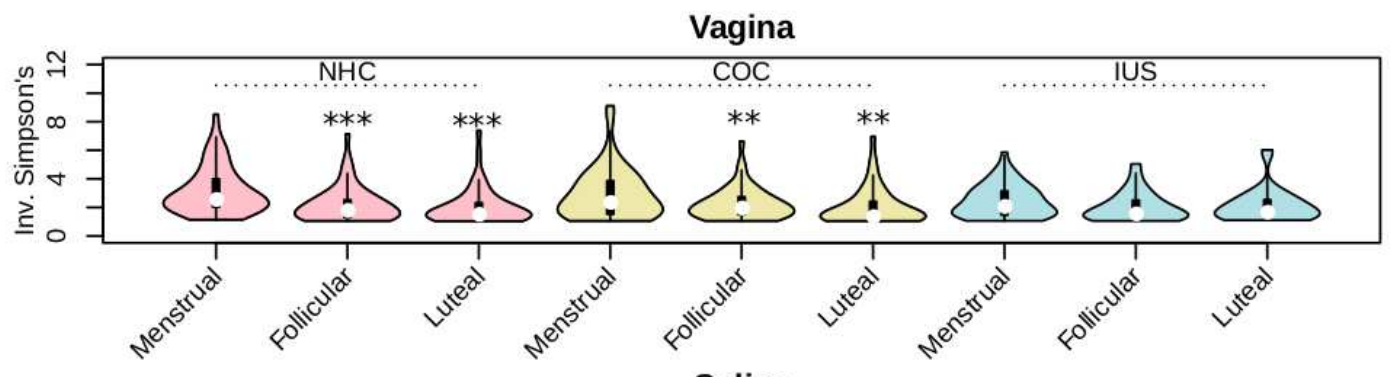

Saliva

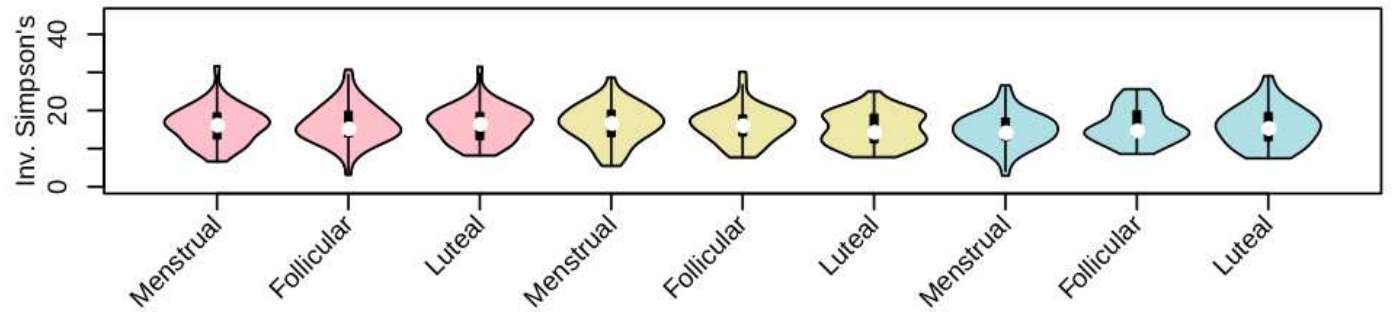

Faeces
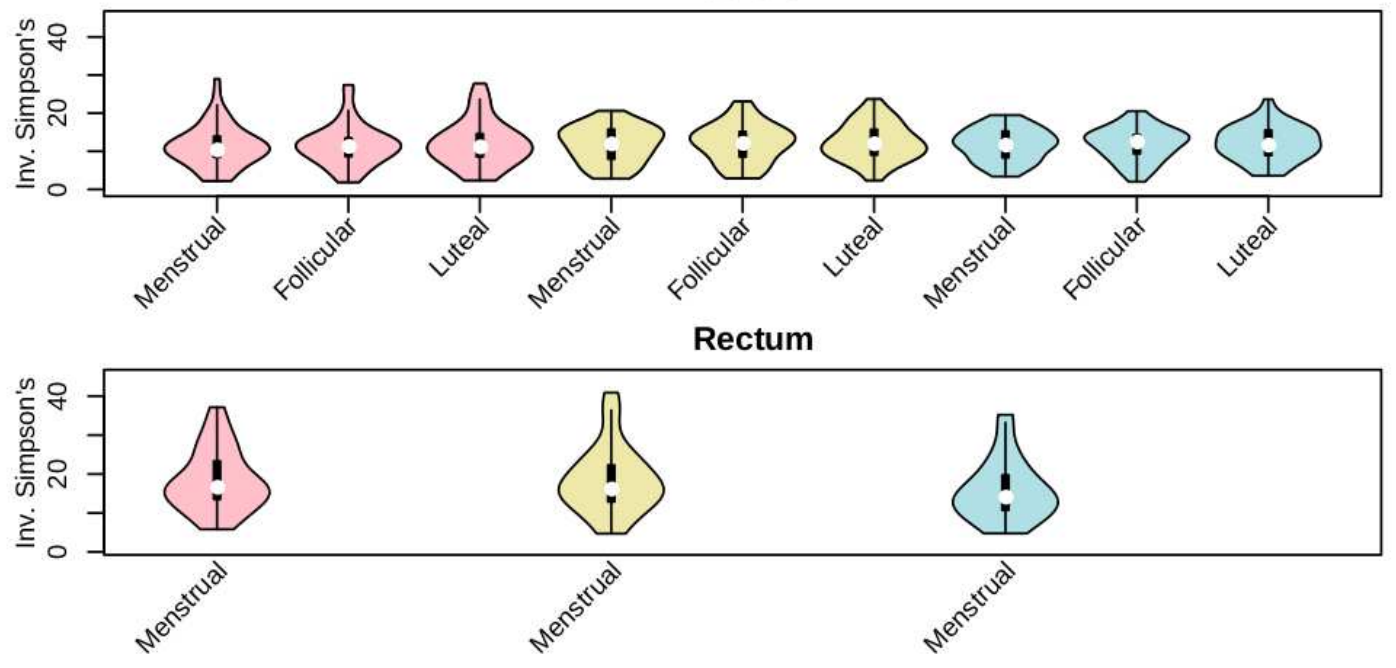

Figure 5: Only vaginal swabs have significant differences in diversity across the menstrual cycle

Violin plots of bacterial diversity (Simpson's inverted index) for vaginal, saliva, faecal and rectal samples. In each panel, samples from women using NHC are depicted in pink, COC in yellow and LNG-IUS in blue. The phase of the menstrual cycle is given in the $\mathrm{x}$-axes. ${ }^{* *} \mathrm{p}<0.01 * * * \mathrm{p}<0.001$ 


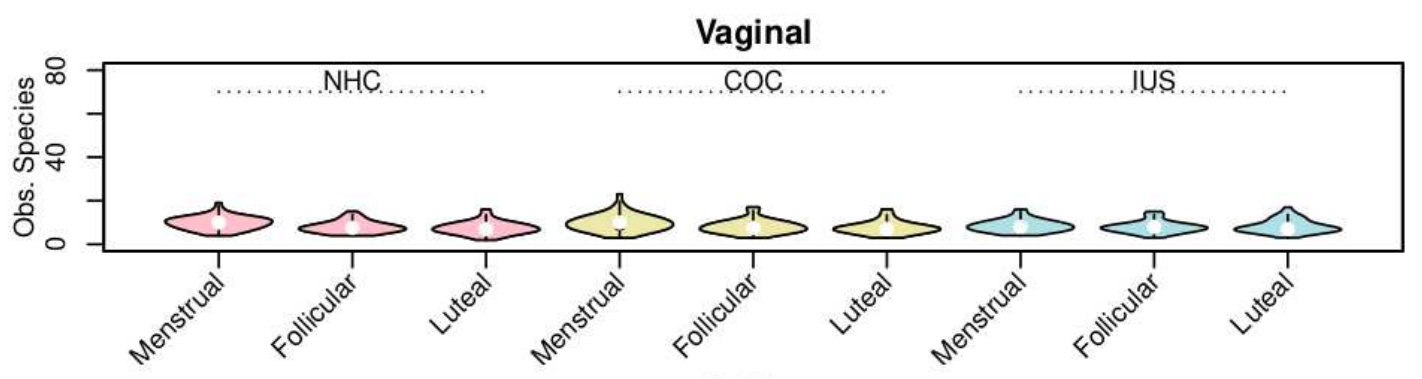

Saliva
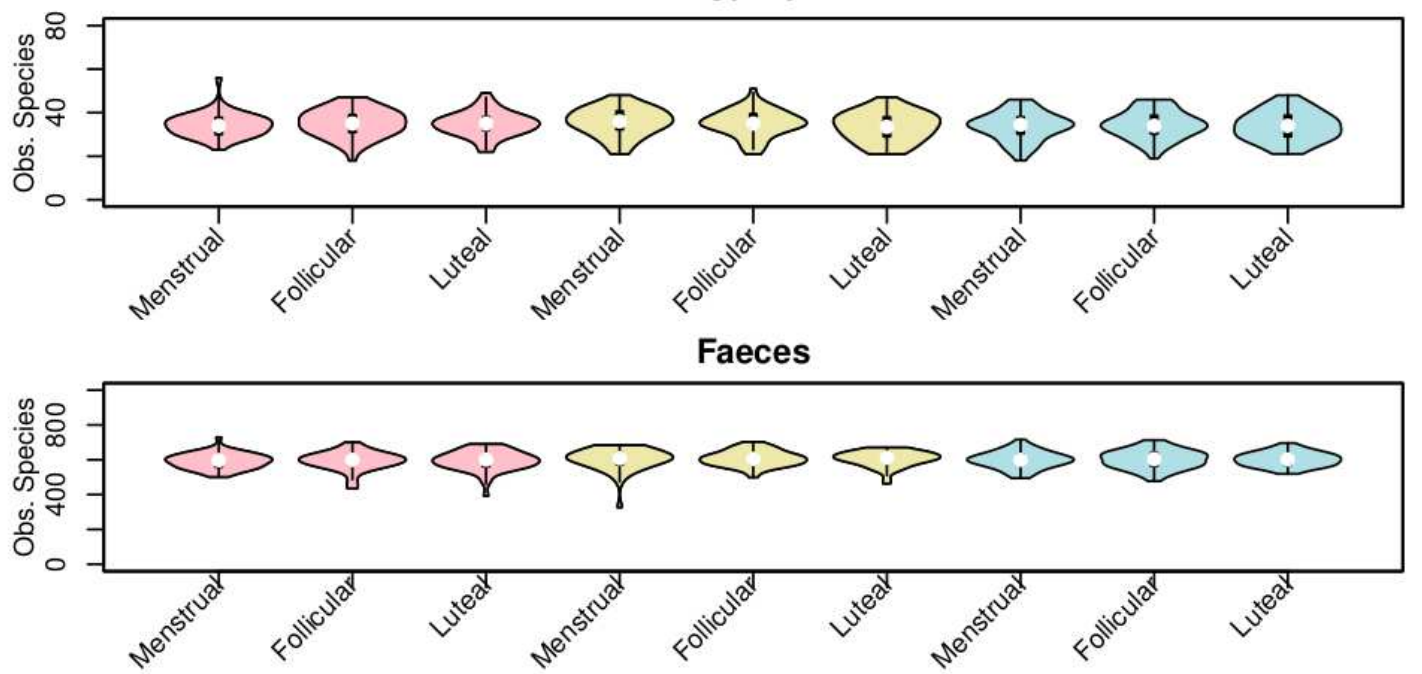

Rectal

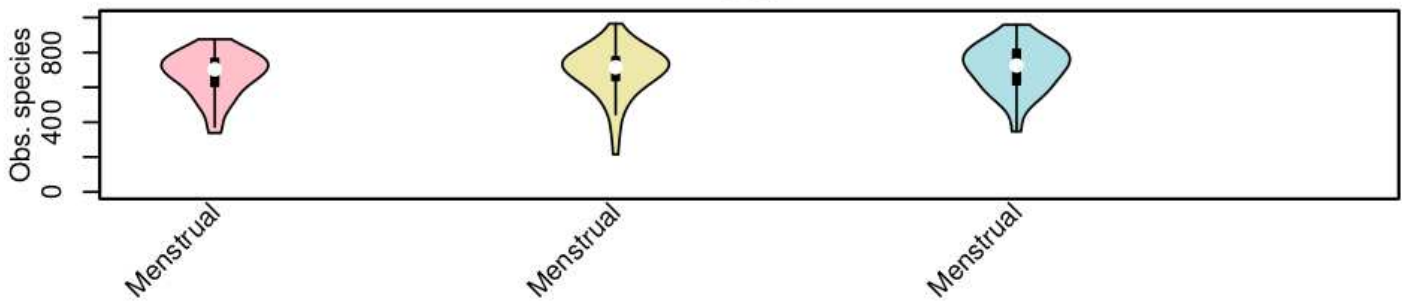

Figure S2: Richness is highest in faeces and rectal samples, and lowest in vaginal samples

Violin plots of bacterial richness (Chao1 index) for vaginal, saliva, faecal and rectal samples. In each panel, samples from subjects not using hormonal contraception are depicted in pink, combined oral contraceptives in yellow and levonorgestrel intrauterine system in blue. The phase of the menstrual cycle is given in the x-axes. 


\section{Sample to sample distance (beta-diversity)}

Samples from each body site are clearly separated from each other (fig. 6), despite certain genera being abundant in two or more sites (fig. S3). Within each body site, the separation between samples is not driven by contraceptive or phase of the menstrual cycle (fig. 7). Within a given individual and body site, distances between samples are on average significantly lower than between individuals ( $<<10 \mathrm{E}-15$ for all body sites; fig. S4).

For the vaginal samples, a clear cluster structure exists, with samples being dominated by one of Lactobacillus crispatus, Lactobacillus iners, Gardnerella vaginalis or Prevotella spp. (fig. 8). None of the other body sites present equally clear clustering (fig. S5). The clustering structure observed for the vaginal samples is not clearly reflected on the other body sites (fig. S5b-d). There is a statistically significant but weak correlation of the sample-to-sample distance between individuals of rectal samples compared to vaginal samples (Mantel's test on Spearman's correlation. $\mathrm{r}=0.058, \mathrm{p}=0.009$; rectal against faecal, $\mathrm{r}=0.26, \mathrm{p}=$ 0.001; other comparisons n.s.; all p adjusted). 


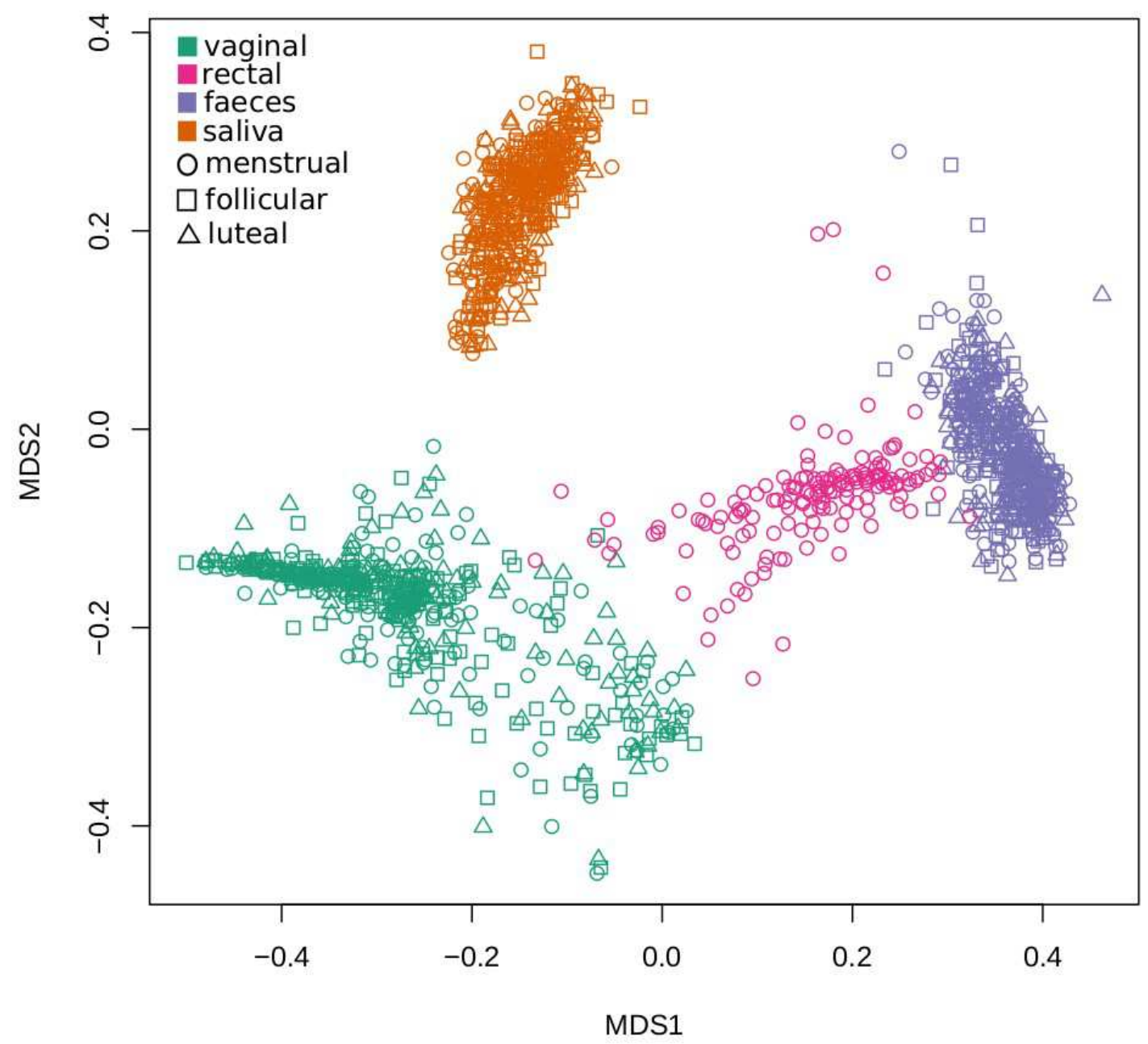

Figure 6: Samples separate primarily by sample type, and not by phase of the cycle

Scatter plot of a 2-dimensional non-metric multidimensional scaling (NMDS) of distances between samples (Bray-Curtis). Each colour represents a body site, and sampling time-point is shown in the shape of the points. 

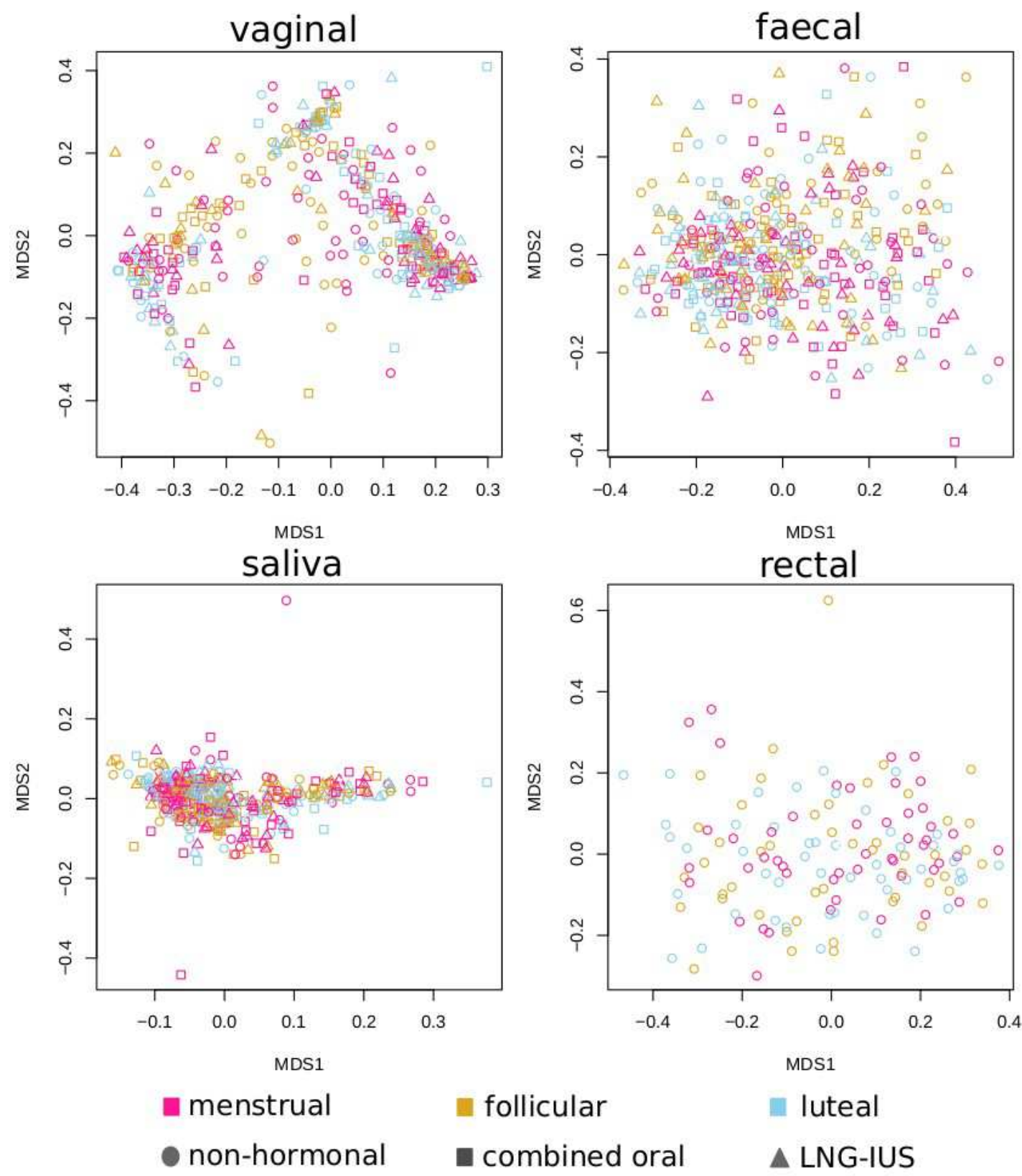

Figure 7: Menstrual cycle and hormonal contraceptive usage are not main drivers of sample separation

Scatter plots of 2-dimensional non-metric multidimensional scaling of distances between samples for vaginal samples, saliva, faecal and rectal samples. None of the body sites presents a clear clustering structure, nor do samples segregate by phase of the menstrual cycle nor contraceptive. Vaginal samples present a striking triangular separation structure. 
L. crispatus

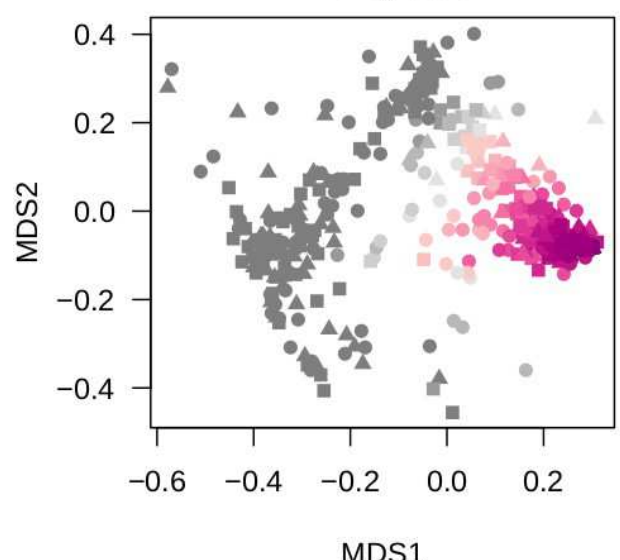

G. vaginalis

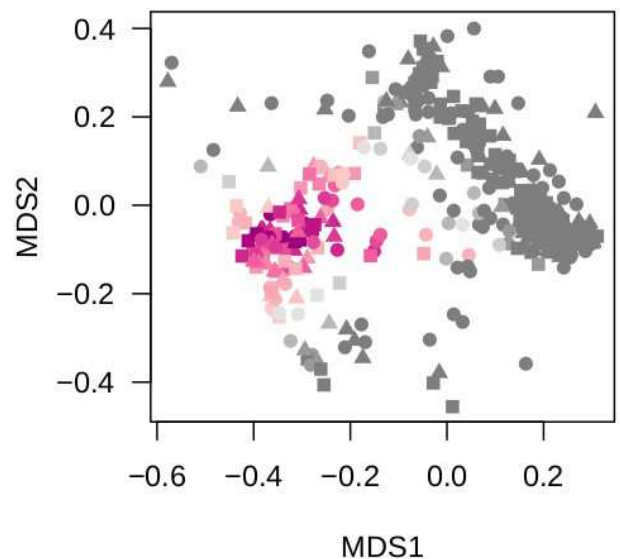

L. iners

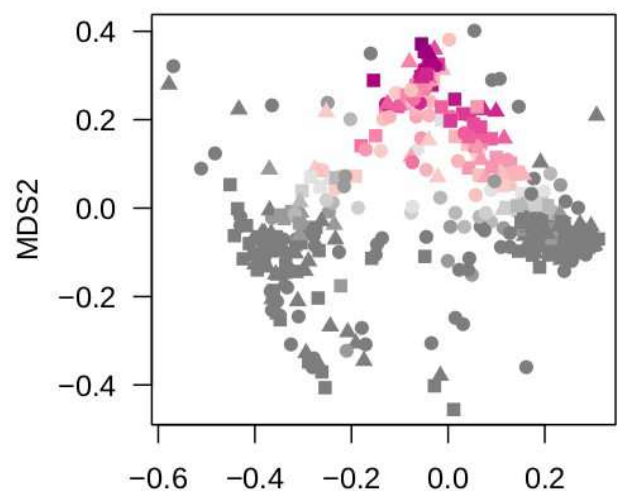

MDS1

Prevotella spp.

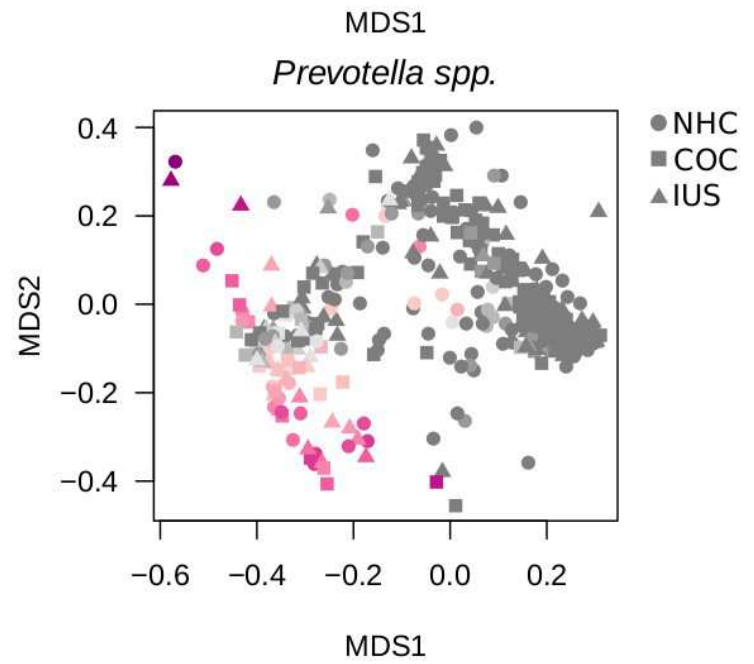

$\% \operatorname{taxon}$

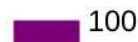

75

50

25

0

Figure 8: Vaginal samples are almost exclusively dominated by one of four taxa The same scatter plot of 2-dimensional non-metric multidimensional scaling of distances between vaginal samples from figure 6 is presented again with a different annotation. In each panel, the relative abundance of one key taxon is depicted in colour scale, from 0 (gray) to 100\% (purple): Lactobacillus crispatus, Lactobacillus iners, Gardnerella vaginalis and Prevotella spp. The first three of these taxa are more or less mutually exclusive, while Prevotella spp can be found in mixtures with other taxa, but most prominently with $G$. vaginalis. 


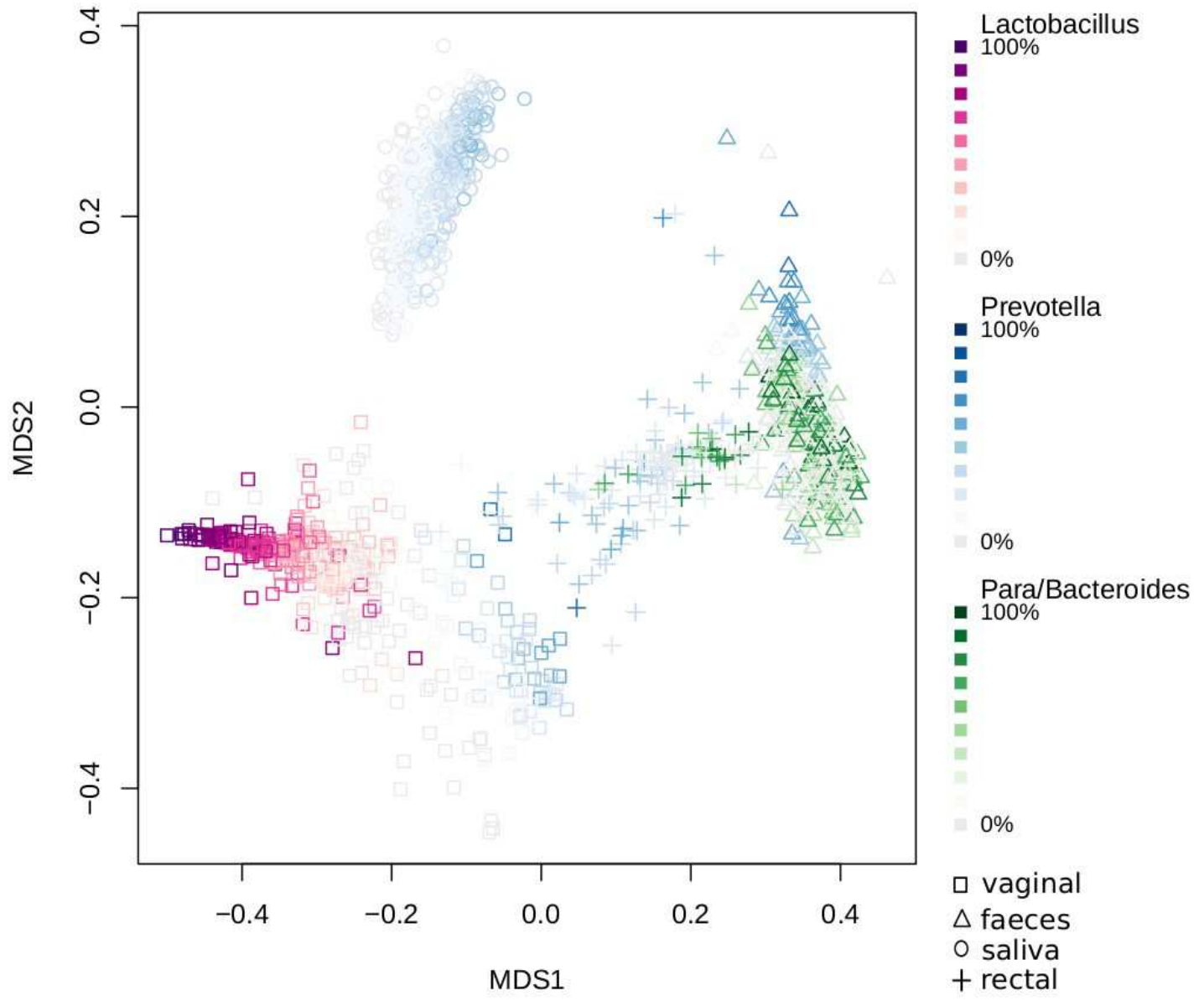

Figure S3: Highly diverse vaginal samples are closer to rectal swabs than Lactobacillus-dominated samples

Scatter plot of a 2-dimensional non-metric multidimensional scaling of distances between samples, same ordination as figure 6. Body sites are depicted by shape. Four key genera are highlighted by colour gradients. 

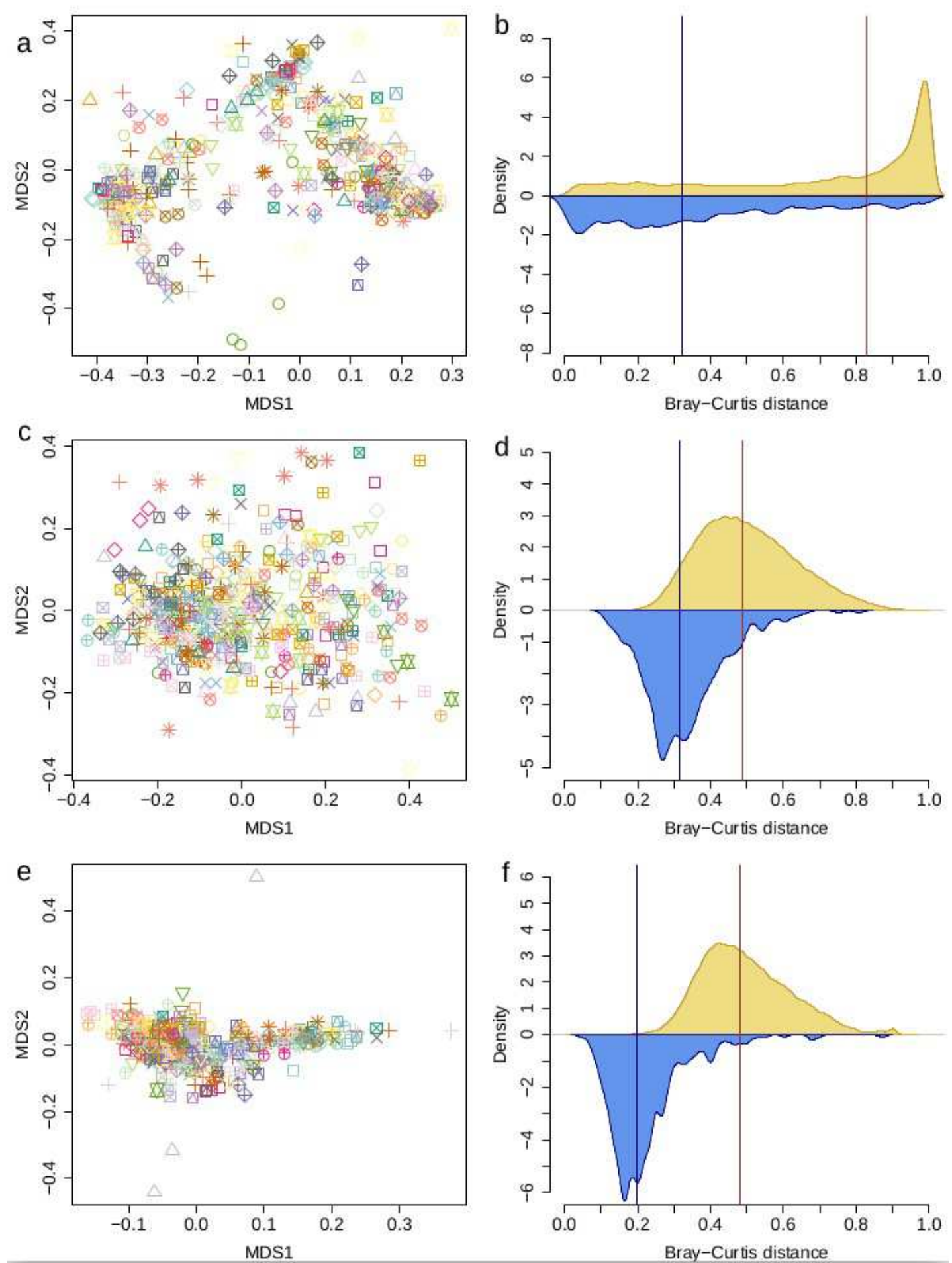

Figure S4: Samples from the same individual can present large distances, but are on average more similar than samples from different individuals

Panels a, c, and e present scatter plots of 2-dimensional non-metric multidimensional scaling of distances between samples for (a) vaginal samples (c) saliva (e) faeces. Points with the same combination of colour and symbol come from the same individual. Panels b, $\mathrm{d}$ and $\mathrm{f}$ quantify the Bray-Curtis distance between samples from the same individual (yellow) or different individuals (blue). The mean of each density distribution is depicted in brown, for within-individual distances, or dark blue, for distances across individuals. 

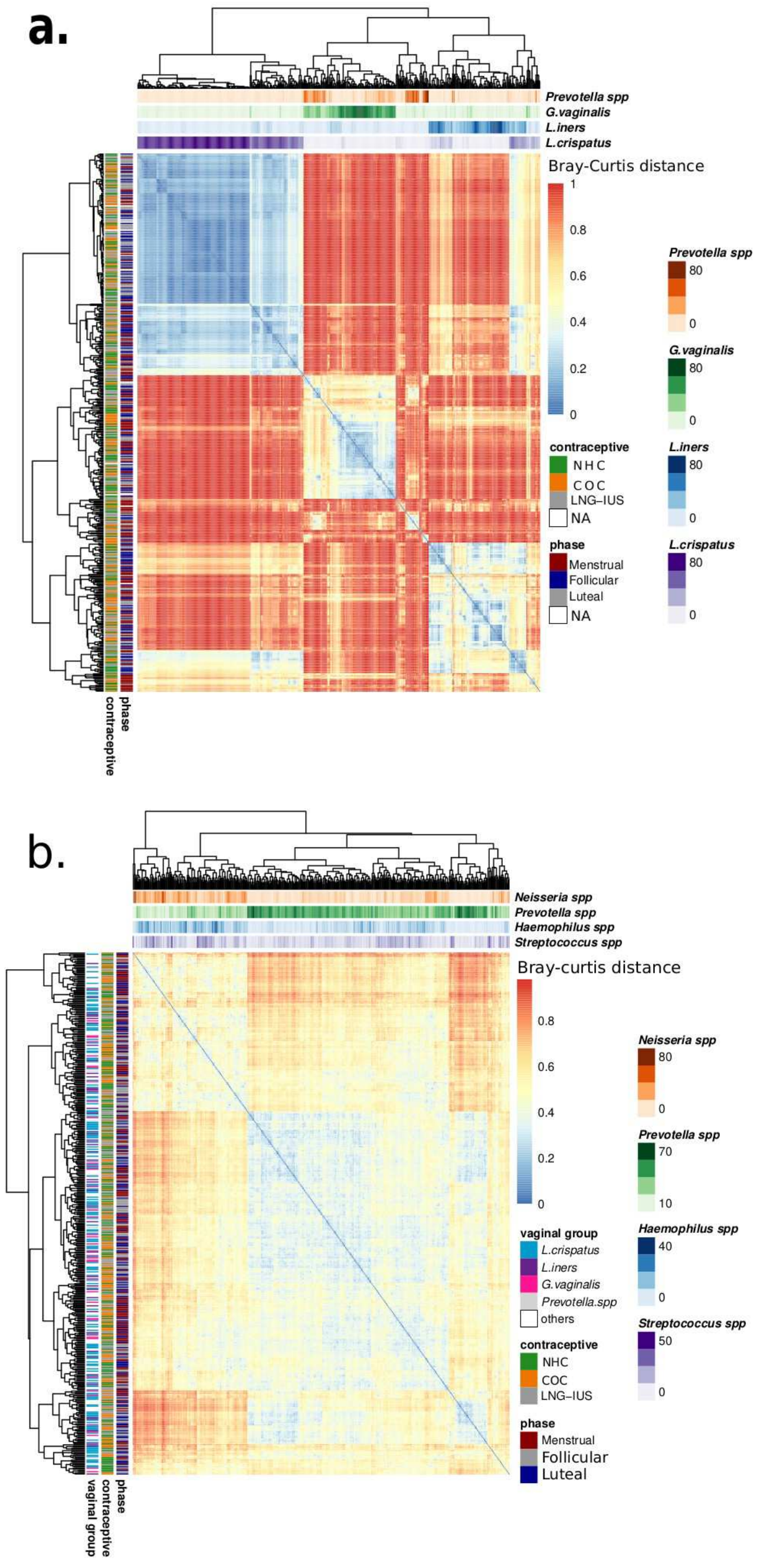

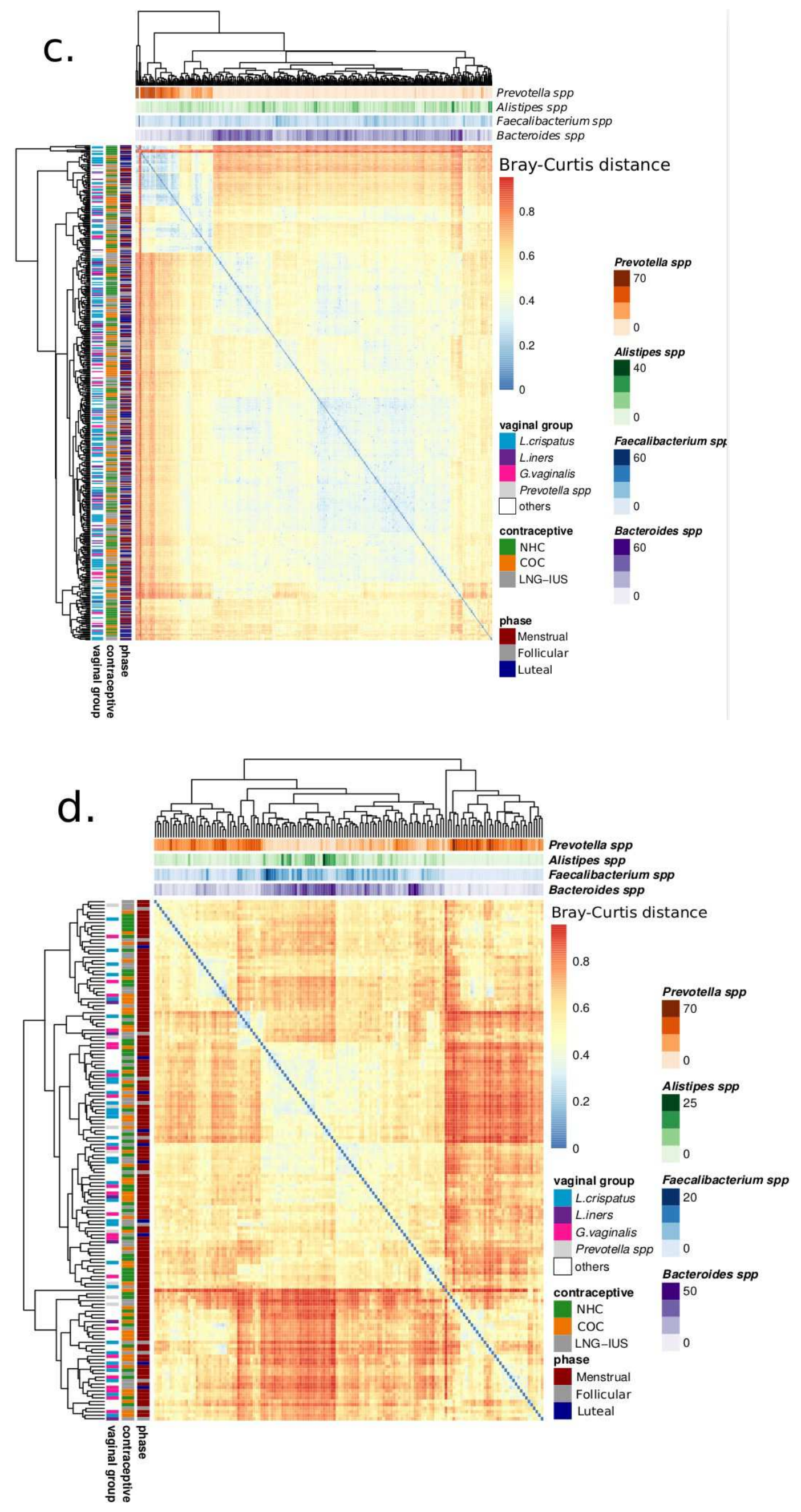
Figure S5: Saliva, faecal and rectal samples do not present the clear clustering found in vaginal samples

Heatmaps showing Bray-Curtis dissimilarity between samples (from dark blue, identical samples, to red, no similarity). Samples are clustered by average linkage. The four rows on top of the heatmap show the relative abundance, in percentage, of four key taxa for each compartment. Columns on the left of each heatmap show the individual's contraceptive method as well as the phase in the menstrual cycle when the sample was taken. For saliva, faeces and rectal samples, the dominant species in the corresponding vaginal sample is also depicted on the left. (a) vaginal swabs (b) faeces (c) saliva (d) rectal swabs

\section{Shifts in microbiome composition during the menstrual cycle}

Shifts between consecutive time-points are most subtle for faecal samples, and most extreme for vaginal samples (fig. 9; $\mathrm{p}<10 \mathrm{E}-15)$. Shifts in their saliva and vaginal microbiome are smallest in the transition from follicular to luteal phase, and similarly high upon entering and passing the menstrual period ( $\mathrm{p}<10 \mathrm{E}-5$ for vaginal samples; $p=0.04$ for saliva). Separating women by their contraceptive group, shifts in the vaginal microbiome are largest for those without hormonal contraception and smallest for LNG-IUS users, even after excluding women without menstrual bleedings (LNG-IUS vs NHC, $\mathrm{p}=0.0005$; COC vs NHC, $\mathrm{p}=0.02$ ). No significant differences between contraceptive groups are observed for saliva or faeces. 

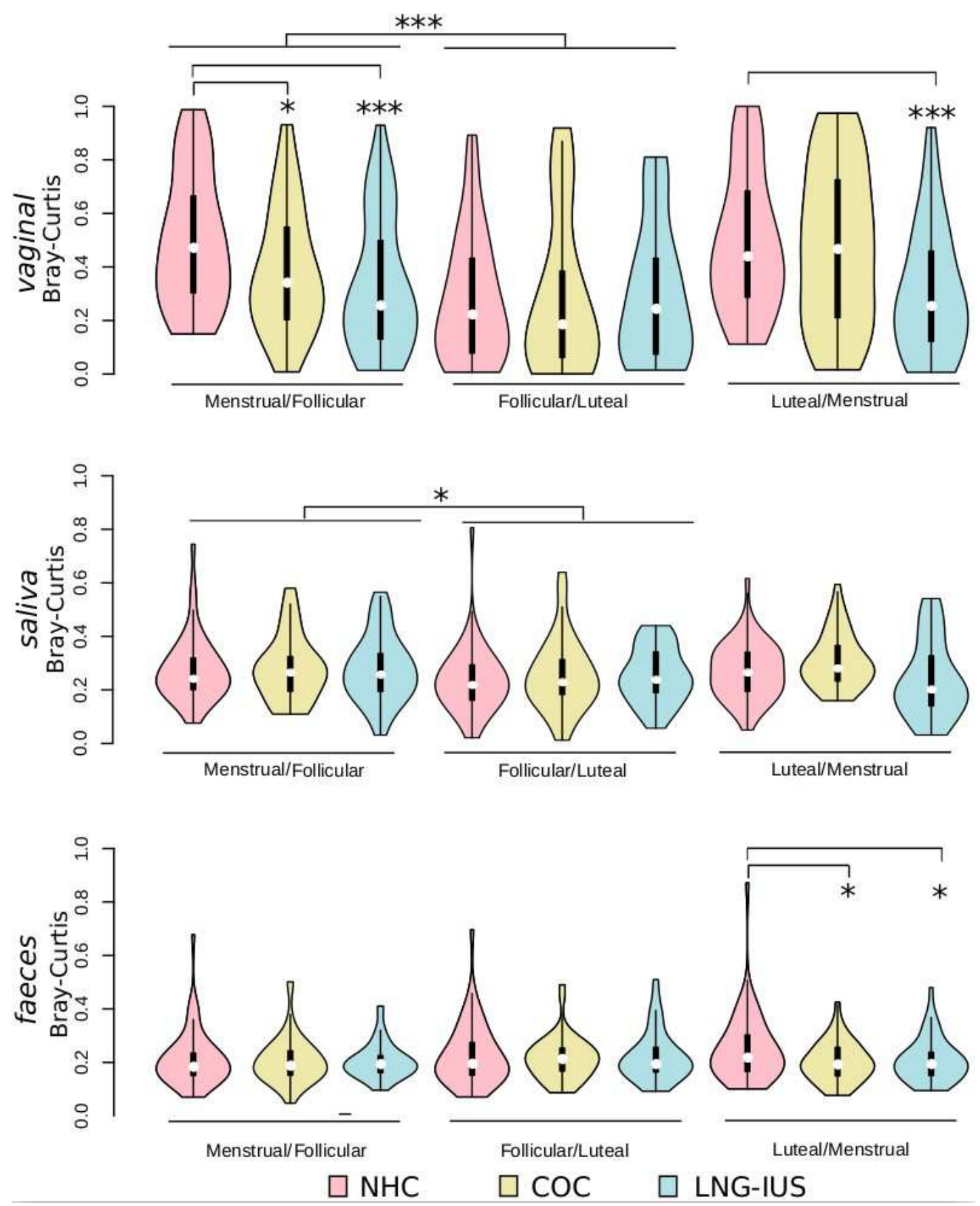

Figure 9: The human microbiome is most variable in the vagina and least stable during the menstrual phase

Violin plots of the within-subject Bray-Curtis sample distance in the transition between each phase of the menstrual cycle. The phase transition is given in the $\mathrm{x}$ axis. Significant differences within a phase transition are all in relation to nonhormonal contraception, and differences between phase transitions are in relation to the menstrual/follicular transition. ${ }^{*} \mathrm{p}<0.05$; ${ }^{* *} \mathrm{p}<0.001$ NHC: non-hormonal contraceptive; COC: combine oral contraceptive; LNG-IUS: levonorgestrel intrauterine system 


\section{Taxonomic composition of vaginal samples and role of sex hormones}

There is no significant difference in the relative abundance of bacterial species between the contraceptive groups. Vaginal samples are dominated by Lactobacillus crispatus, Lactobacillus iners, Gardnerella vaginalis and Prevotella spp. regardless of contraceptive method (Fig. 2). Genus Lactobacillus and L. crispatus expand in abundance during the follicular and luteal phases (Welch's t-test. In the follicular phase $\mathrm{p}=0.0002$ for Lactobacillus and $\mathrm{p}=0.004$ for L . crispatus and in the luteal phase, $\mathrm{p}=$ 2E-6 for Lactobacillus and p = 3E-6 for L. crispatus). Concomitant to this increase in Lactobacillus spp., there is a decrease in Gardnerella spp. (fig. 10).

Four Lactobacillus species are increased in the follicular and luteal samples compared with the menstrual sample, while eight bacterial vaginosis (BV)associated species are decreased (fig. 11). In addition to this, Atopobium vaginae is increased in snus users $(\mathrm{r}=0.026, \mathrm{q}=0.035)$.

Genus Lactobacillus and L. crispatus are positively correlated to serum oestradiol levels ( $\mathrm{r}=0.11, \mathrm{p}<0.001$; fig. 12). Another two Lactobacillus species have significant positive correlations with sex hormones (L. kitasatonis $\sim$ oestradiol, $\mathrm{r}=$ 0.003, $\mathrm{q}=0.041 ;$ L. amylovorus $\sim$ progesterone, $\mathrm{r}=8.5 \mathrm{E}-5, \mathrm{q}=0.013$ ). Women with high levels of $L$. iners have on average higher serum oestradiol levels (n.s.; fig S6). No species in the saliva or faecal microbiome are significantly associated to sex hormone levels after adjusting for the individual. 


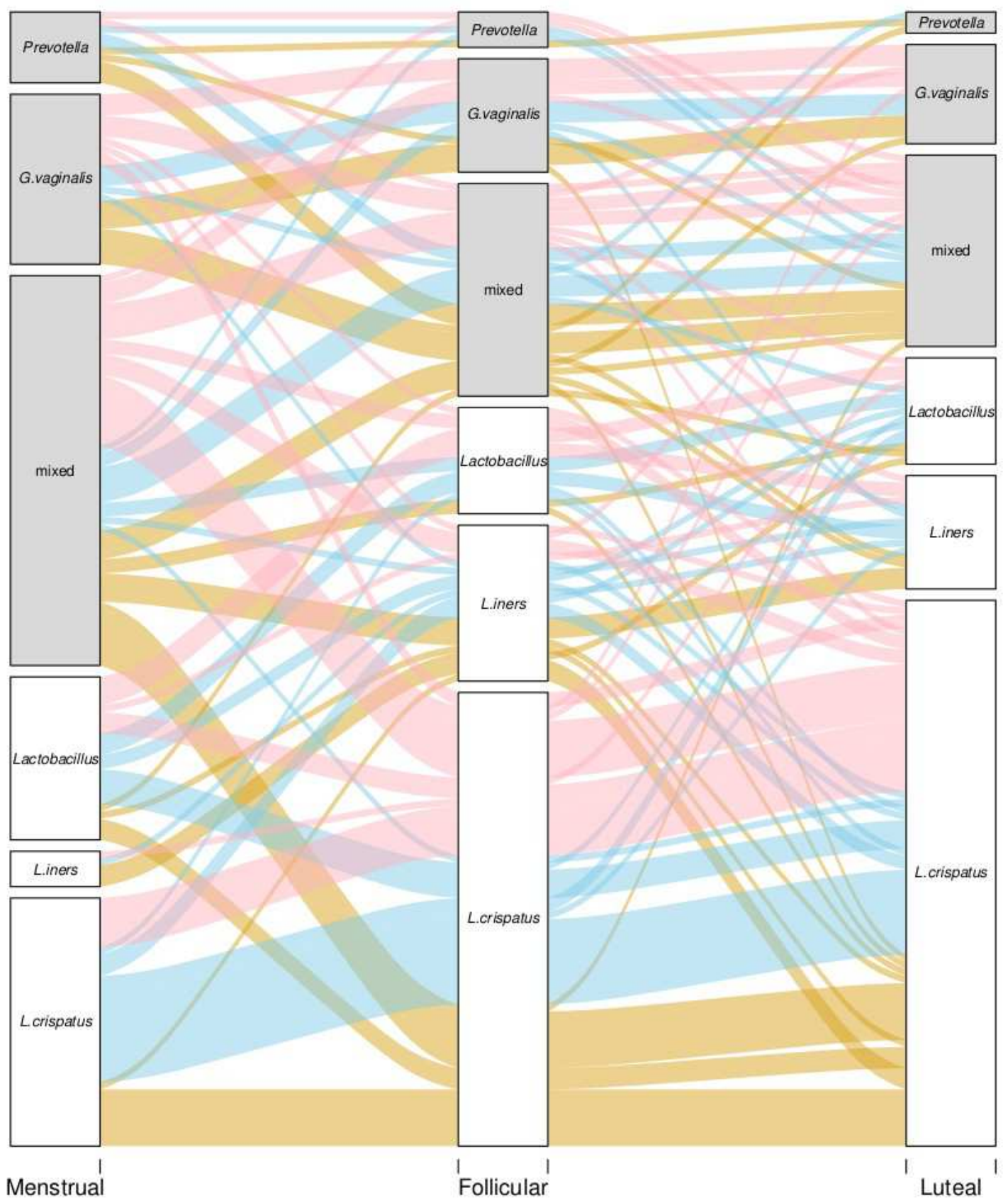

Figure 10: Over the menstrual cycle, the vaginal microbiome of more women becomes dominated by Lactobacillus crispatus.

Alluvial plot showing the fate of each sample across the menstrual cycle.

"Dominance" is defined as $>60 \%$ of reads coming from the same genus or species.

The "Lactobacillus" groups comprises samples with a mixture of Lactobacillus species. Samples lacking a dominant group are classified as “mixed”. The three groups not dominated by Lactobacillus species are shaded grey. The lines depicting the trajectory of each sample are coloured after the contraceptive used by that individual. Pink: non-hormonal contraceptive. Yellow: combined oral contraceptive. Blue: LNG-IUS. 

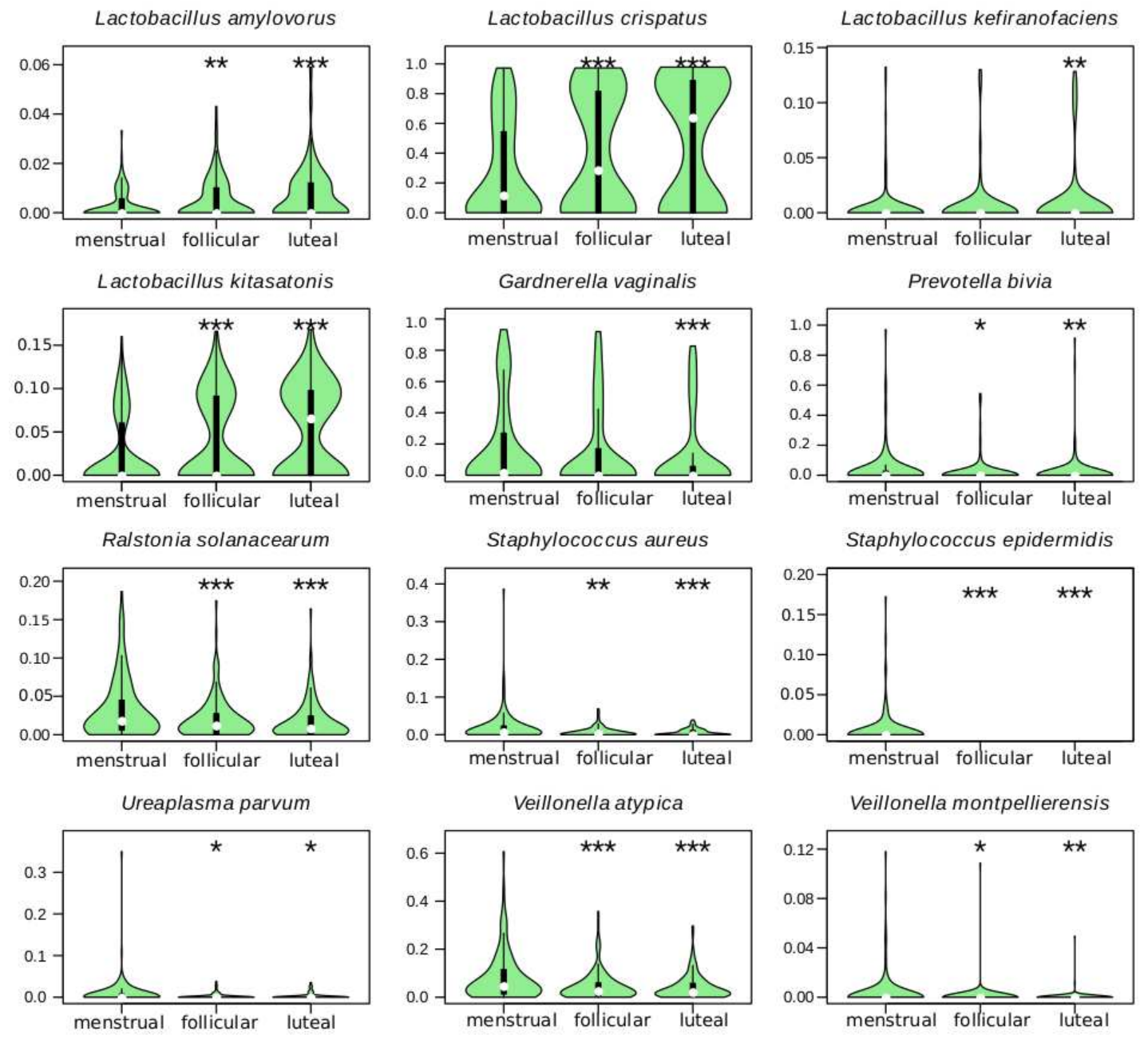

Figure 11: Species whose abundance in the vagina varies over the menstrual cycle

Violin plots showing the relative abundance of species whose abundance is significantly correlated to the phase of the menstrual cycle, adjusted for contraceptive usage and lifestyle variables. Y-axis in each panel is adjusted to show the differences in the data, so scale varies. ${ }^{*} \mathrm{p}<0.05,{ }^{* *} \mathrm{p}<0.01,{ }^{* * *} \mathrm{p}<0.001$ 
Lactobacillus

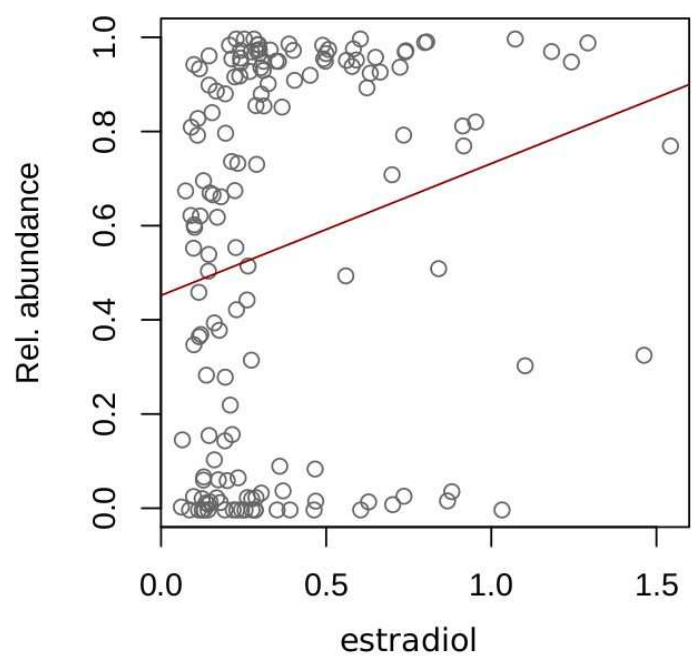

Lactobacillus crispatus

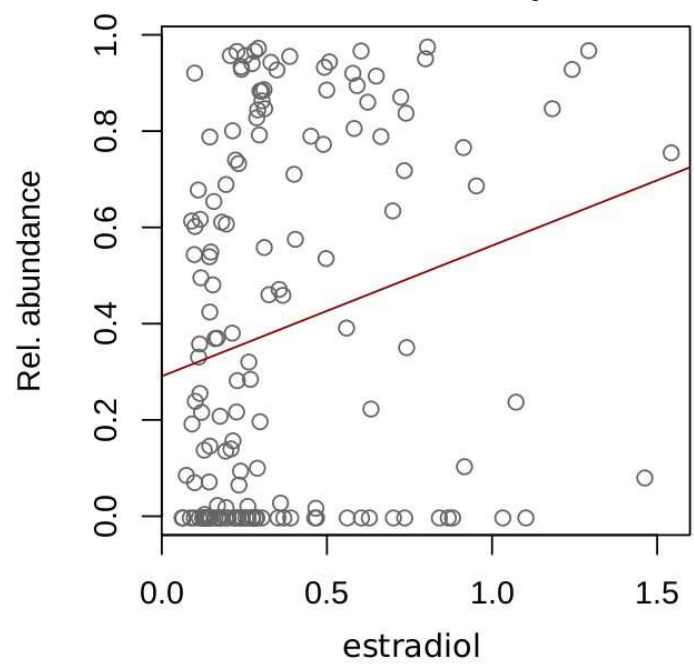

Figure 12: Lactobacillus spp abundance correlates with oestradiol levels Both genus Lactobacillus and the species Lactobacillus crispatus are more abundant in the presence of high oestradiol levels, although some women may present low Lactobacillus spp. abundance regardless of their oestradiol level.

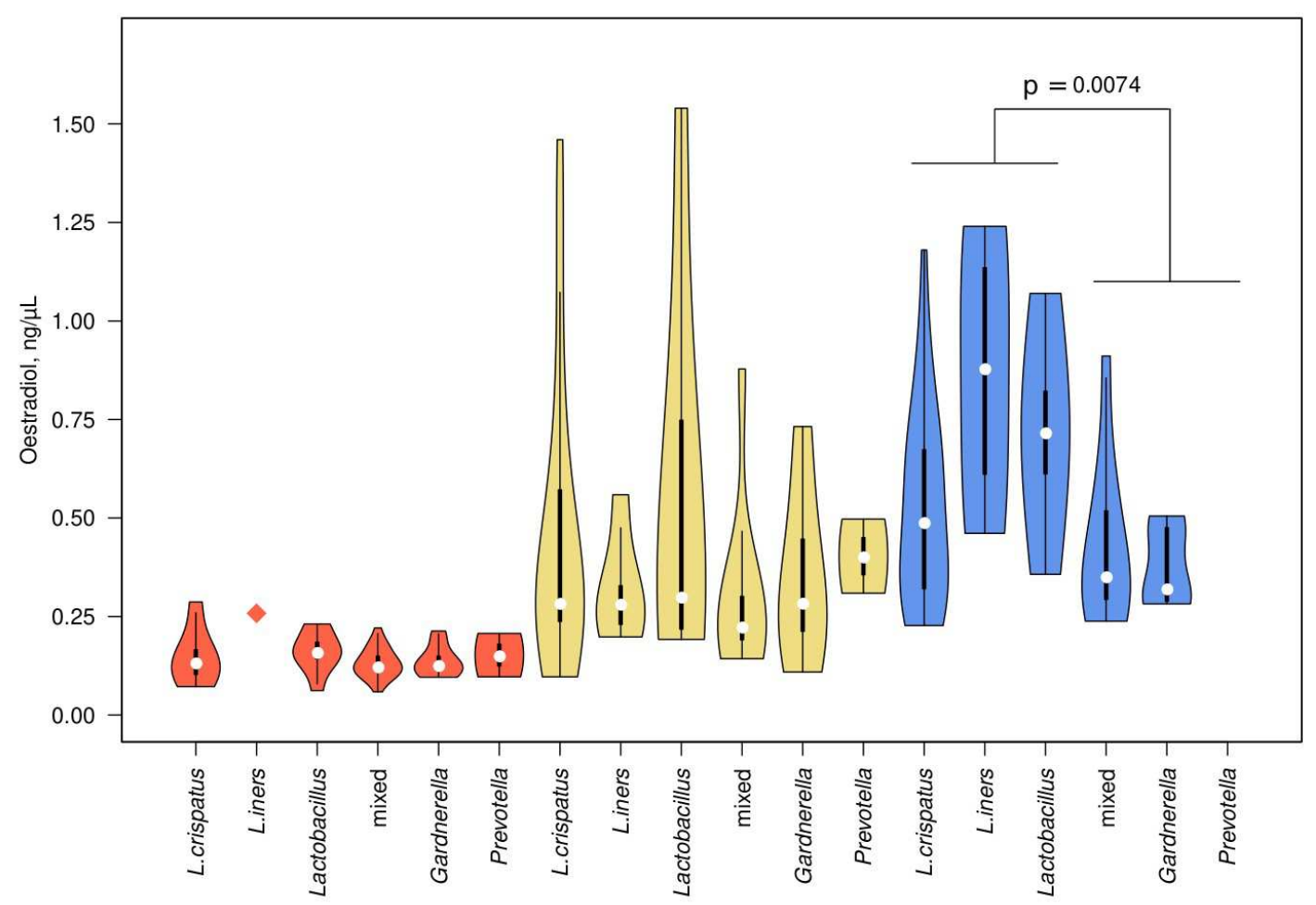

Figure S6: Serum oestradiol levels differ for women with different dominant vaginal communities

Violin plots depicting serum oestradiol levels for women not using hormonal contraception, divided by phase of the cycle and dominant vaginal species. Samples are assigned to a taxonomic cluster if the relevant taxon corresponds to $>60 \%$ of the total gene content. Because the sample size for each group is small, statistical comparisons are done between high Lactobacillus and low Lactobacillus groups. 


\section{Vaginal dysbiosis and sexually transmitted infections}

Most women who present with a dysbiotic vaginal microbiome during menses change to a Lactobacillus-dominated microbiome in the subsequent follicular and luteal phases (fig. 10). During menses, 89 women (58\%) have a dysbiotic vaginal microbiome with less than 60\% Lactobacillus spp. This decreases to $49(32 \%)$ in the follicular phase $(\mathrm{p}=1.3 \mathrm{E}-5)$ and $44(29 \%)$ in the luteal phase $(\mathrm{p}=$ 4.6E-7). Most of this shift comes from women with a mixed microbiome (no species $>60 \%$ abundance) becoming more Lactobacillus dominated, but also from a decrease in Gardnerella vaginalis. No differences in demographic or health questionnaire data are found in women with Lactobacillus-dominated vaginal microbiomes compared with others and in women dominated by BV-associated bacteria compared with others.

A significant positive correlation is observed between the proportion of human DNA in a sample and the Lactobacillus content $(r=0.54, \mathrm{p}=5 \mathrm{E}-35)$. Conversely, typical BV bacteria, such as Gardnerella $(\mathrm{r}=-0.43, \mathrm{p}=2 \mathrm{E}-21)$ and Prevotella $(\mathrm{r}=-0.53, \mathrm{p}=\mathrm{E}-34)$ are negatively correlated to the proportion of human DNA in a sample.

Sexually transmitted diseases are identified in two participants. One woman not using hormonal contraception was diagnosed with Chlamydia trachomatis and Neisseria gonorrhoea shortly after providing her samples. These bacteria are also found in relatively low amounts (0.5-1.5\% of reads) in all her vaginal samples which also presented with relatively large amounts of Gardnerella (20-30\%) and other bacteria such as Prevotella and Veillonella. Another woman, who had completed treatment for Chlamydia trachomatis two months prior to her enrolment in the study has up to $2.5 \%$ of the DNA in her vaginal swab from this species. Her vaginal samples are dominated by $L$. iners and $L$. jensenii, but also present about 10-20\% of Ralstonia solanacearum and Veillonella atypica. 


\section{Discussion}

Herein we present the largest investigation of human microbiomes by shotgun sequencing to date. We investigate four body sites at three points of the menstrual cycle in 160 healthy Caucasian women of reproductive age using three different contraceptive regimens (fig.13).

\section{The microbiome across body sites, contraceptive groups and phases of the menstrual cycle}

In a Danish population of young, healthy women the type of contraception did not associate with microbiome composition in three body sites: rectum, faeces and saliva, at any of the three timepoints in the menstrual cycle (menstrual, follicular and luteal phases). Levels of oestradiol and progesterone did also not associate with microbiome composition in these body sites. This knowledge is novel and important as hormonal contraception is widely used during early reproductive years, often over several years [45]-[47], before attempting pregnancy.

The hypothesis that different body sites are interconnected and affect each other is here supported by significant sharing of strains across body sites (fig. 4). This connection might be due to other systematic mechanisms (apart from female sex hormones) in the individual, such as genetics, immunological, hormonal and even priority effects. It could also simply be a physical connection, with microorganisms traveling alongside food through the digestive system (oral/faecal/rectal), sexually (oral/vagina/rectal) or due to anatomical proximity (vaginal/rectal/faecal). However, while highly significant, the correlations are small (0.1-0.27) and one body site cannot be sampled as a proxy for another site.

Body site is by far the most prominent feature separating microbiome samples (fig. 6) [14], [48], [49]. We included the rectum, a body site that is not often sampled and observe that it shares many characteristics with faeces (fig. 4) [50], [51], but it is also unique. The rectal microbiome has previously been suggested to be a reservoir of bacteria that can readily colonise the vagina [52]. This is partially confirmed here in the observation that Lactobacillus strains found in the rectum are significantly more frequent in the vagina of the same individuals, as previously reported (fig. 4) [53]. Importantly, no such overlap occurs between faecal and vaginal samples, which makes inappropriate wiping routines quite unlikely to explain the shared strains between rectum and vagina. A previous study found a significantly shorter ano-vaginal distance in women diagnosed with bacterial vaginosis (BV) compared with women without BV, and suggesting this as a possible anatomical explanation for the development of dysbiosis [54]. This strain-level 
analysis was only possible due to our use of shotgun metagenomics, since it would not have been possible using 16S rRNA analysis or other marker genes.

The composition of the saliva and faecal microbiomes were largely unaffected by menstrual cycle and contraceptives in our study (fig. 3). Accordingly, no specific bacteria in these body sites could be connected to serum levels of oestradiol or progesterone. However, the saliva microbiome seems to be least stable during menses (fig. 9) as previously reported [36].

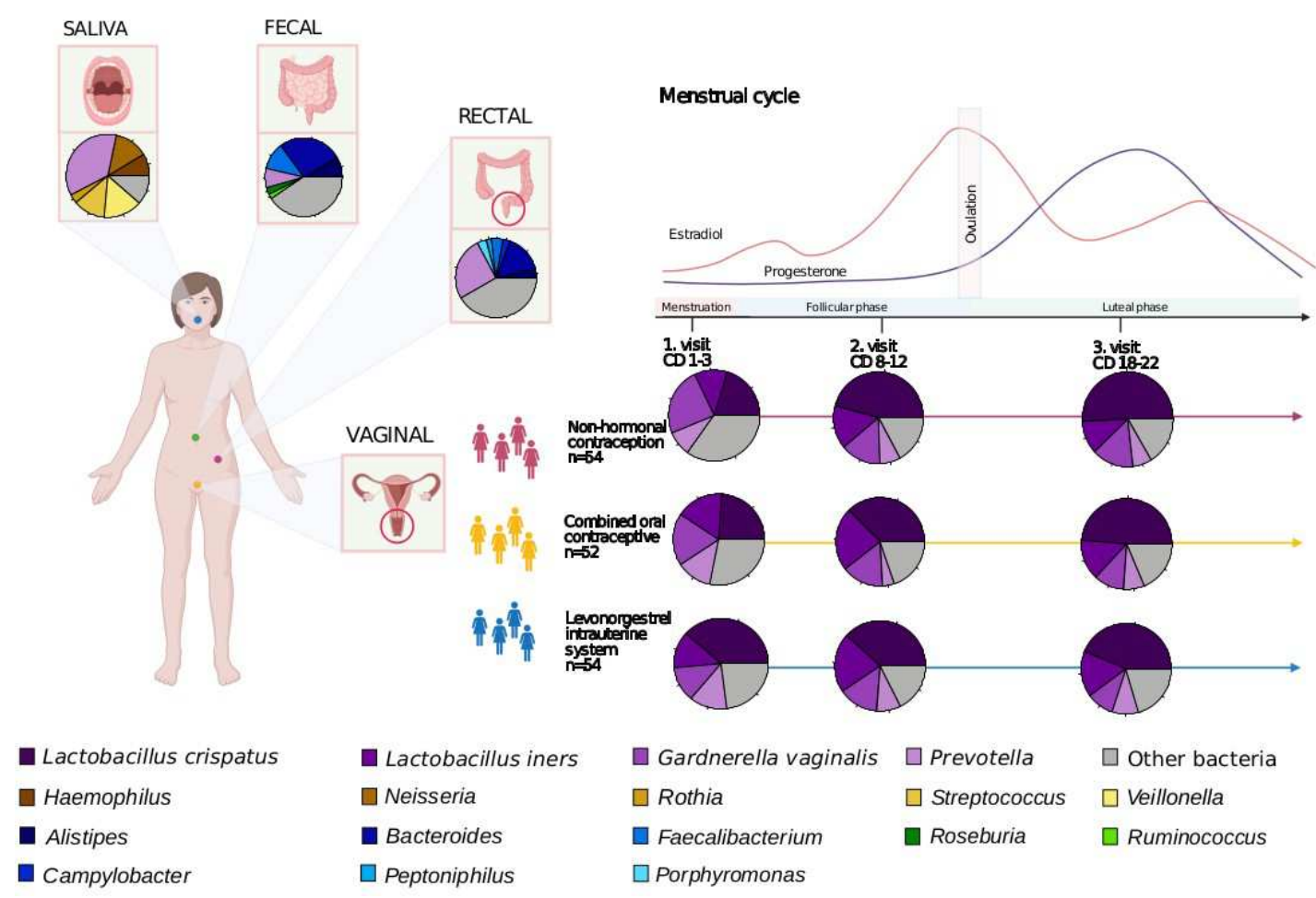

Figure 13: Schematic representation of the sampling scheme and taxonomic profiles

Over 50 women were recruited using one of three contraceptive regimens: NHC (non-hormonal contraceptives), COC (combined oral contraceptives) and LNG-IUS (levonorgestrel intra-uterine system). Included women were sampled at the hospital during the menstrual phase (cycle days 1-3), follicular phase (cycle days 8-12) and luteal phase (cycle days 18-22). Blood samples, saliva and rectal swabs were collected at the hospital; vaginal swabs and faeces were collected by the women at home. Women on LNG-IUS with oligo-/amenorrhoea started their sampling at a random day. 


\section{The vaginal microbiome responds to female sex hormones}

Differences according to contraception method were found in the vaginal microbiome (fig. 13). Women not using hormonal contraceptives (NHC group) had a significantly larger shift in the composition of their vaginal microbiome across the menstrual cycle, compared with the COC and LNG-IUS groups; the latter remained the most stable throughout the menstrual cycle, regardless of whether they had menstrual bleedings or not (fig. 9).

We find an increase of Lactobacillus species and a decrease of eight $\mathrm{BV}$-associated species in the follicular and luteal phases (fig. 11). The cause of the increased diversity in the vaginal microbiome during menstrual bleeding has been debated: are differences observed during menstruation due to hormonal changes or physiological changes with menstrual blood flowing through the vagina leaving iron-sources for Gardnerella spp. [55] or merely the menstrual hygiene products used. Several previous studies state that low oestradiol levels at the time of menstruation are responsible for low glycogen deposition and thereby lower Lactobacillus presence, resulting in higher $\mathrm{pH}$ which allows growth of anaerobic BV-related bacteria[56]. Further evidence of the association between oestradiol and lactobacillus dominance is provided by studies of menopausal women with vaginal dysbiosis returning to a Lactobacillus dominated microbiome after commencing hormone replacement therapy [27], [57], [58]. Additionally, dysbiosis have been observed in girls before puberty (before oestradiol rise)[26], [59], [60] and in transgender men with oestrogen suppression[61], while the opposite is observed during pregnancy, concomitantly with high levels of oestrogen [13], [62]. Here, we find a direct correlation between oestradiol levels and Lactobacillus spp. in general and with L. crispatus specifically. While previous studies have argued for this association[9], [32], this is, to our knowledge, the first direct measurement of sex hormones in relation to microbiome composition.

Song et al. raise the question whether it could be the decline in progesterone rather than oestradiol before the menstrual bleeding that is responsible for the high diversity during menstruation [32]. [55]. In this study we find increased diversity in the vaginal microbiome during menstruation in all three contraceptive groups (fig. 5). They all have a drop in the level of oestradiol/oestrogen synthetics before menstruation, but the COC group does not ovulate and has no large progesterone shifts prior to menstruation, indicating that the dysbiosis is not driven by progesterone dynamics. The direct association between measured oestradiol levels and vaginal microbiome composition support that the high diversity observed during menses is mainly due to oestradiol withdrawal before menses (fig. 12). We 
can, however, not rule out that the bleeding itself could contribute to the high diversity, as LNG-IUS users with no or little menstrual bleeding despite their underlying cycle and ovulation seem to have the most stable vaginal microbiome (fig. 5). This stability could perhaps also be mediated through changes in the composition of the vaginal mucus because of the local effect of the progestinreleasing device.

We found the vagina to be the only body site with a clear clustering with four main clusters, namely: dominated by a) Lactobacillus crispatus, b) Lactobacillus iners, c) Gardnerella vaginalis and d) Prevotella spp, irrespective of contraception use (fig. 8). This is in contrasts with previous findings by Ravel et. al who identified five specific community state types (CSTs) in the vaginal microbiome of four ethnic groups of women, including a white north American group, based on 16S rRNA sequencing. Four of these CSTs were dominated by different Lactobacillus species (L. crispatus, L. gasseri, L. iners and L. jensenii) leaving the remaining group IV as the diverse group[9], which was later expanded into two clusters (IV-A and IV-B)[37]. In our cohort, while L. gasseri and L. jensenii are both detected, these species are not sufficiently abundant to form separate clusters. These two groups were the smallest in Ravel's pioneering work, and were also found in low prevalence in a previous cohort of Nordic women [63]. Unlike most previous work in the vaginal microbiome, here we have used metagenomic shotgun sequencing instead of 16S rRNA gene sequencing. While the large proportion of human reads did not allow us sufficient sequence depth to assess the bacterial functional potential of vaginal swabs, we could nevertheless make a deeper taxonomic analysis. In addition to this, we have measured key sex hormones at each sampling time point. Previous work has used reference values for sex hormones in relation to cycle day [9], [32]. While our analysis may present a less clear picture of the complex interaction between hormones and microbes, it is nonetheless a more accurate picture.

\section{Vaginal dysbiosis}

Vaginal dysbiosis with a microbiota dominated by BV-associated bacteria, is not associated to contraception use, but is found in $40 \%$ of samples in our cohort, most notably during the menstrual phase (58\% of samples, fig. 10). A previous study found in a cohort of healthy women without BV-symptoms that $27 \%$ presented with vaginal dysbiosis [9], similar to what we find in the follicular and luteal phases (32-29\%). None of our metadata are associated with BV-related species in the vagina, apart from Atopobium vaginae which is significantly increased in snus users. There are no correlations with other related variables (smoking, 
alcohol, number of intercourses) that could be suspected to be confounding of using snus or related to BV. Recent studies conclude that the presence of Atopobium vaginae together with Gardnerella vaginalis provide high predictability of BV [64], [65]. Considering that only few women reported that they used snus $(n=14)$, this could be a coincident finding. Only two women reported current/recent sexually transmitted disease (Chlamydia trachomatis and Neisseria gonorrhoea) which was reflected in the sequencing data. Perhaps a selection bias of non-dysbiotic microbiomes may have been introduced since three women using antimicrobials or lactic acid suppositories during the study were excluded, nevertheless, the cohort can still be considered healthy.

No longitudinal shifts in the cluster structure in the vaginal microbiome was observed during the menstrual cycle regardless of contraceptive use (fig. 10), albeit most women dominated by Gardnerella vaginalis or Prevotella during menses shifted towards a Lactobacillus dominated composition throughout the cycle. Other studies have correlated dysbiosis with reproductive complications [8], [10], [13] and future studies need to explore if those with vaginal dysbiosis throughout the menstrual cycle constitute a different group compared with those with dysbiosis only during menstruation. It can be speculated that reproductive complications are only associated with women who, despite an increase in oestradiol, maintain a dysbiotic vaginal microbiome throughout the menstrual cycle. Another interesting finding in this study is the observation that the proportion of human DNA in a vaginal sample is negatively correlated to typical BV-associated bacteria, such as Gardnerella vaginalis and Prevotella spp and, conversely, positively correlated with the Lactobacillus content. This may be due to the shedding of clue cells (Amagshie et al 2017) with a high bacterium to human DNA ratio, and/or due to the thick, bacteria-dense biofilm formed on top of the epithelial surface by BV-associated bacteria [66]. This is relevant for future work using shotgun sequencing, since Lactobacillus spp. dominated samples will be sequenced less deeply unless human DNA removal is performed before sequencing.

This is the first large scale shotgun investigation of the microbiota composition across four body sites in a healthy female Caucasian cohort. Another strength is the comparison between a natural menstrual cycle and cycles affected by hormonal contraception use, as well as real-time measurements of endogenous sex hormone levels. The extensive questionnaire data regarding the women's prior and current health, lifestyle and sex life enable us to explore and adjust for potential impact on the microbiota compositions across body sites. A limitation is the homogeneity of the cohort which makes it impossible to investigate the impact of 
ethnicity and low socio-economic status. Another limitation is that only three time points in the menstrual cycle are investigated in this study leaving day-to-day variations unexplored.

\section{Conclusions}

The type of hormonal contraception does not significantly associate with the microbiome composition in the vagina, faeces, rectum or saliva in healthy young women. This is a welcome finding considering the widespread and prolonged use of these highly efficient contraceptive methods. The menstrual cycle is, however, a major confounding factor for the vaginal microbiome, with large shifts in composition occurring around menstruation. These changes are most pronounced in women with a natural cycle, compared with COC and LNG-IUS users. The time point in the menstrual cycle should be considered when analysing the microbiome of women of reproductive age, since stratifying by vaginal dysbiosis status during menstruation might be misleading. This is the first study to confirm by direct measurements of oestradiol, a correlation of high oestradiol levels with the presence of Lactobacillus crispatus during a natural cycle, adding evidence of a possible hormonal mechanism for the maintenance of this desirable microbe. 


\section{List of abbreviations}

NHC - non-hormonal contraceptives; COC - combined oral contraceptives; LNGIUS - levonorgestrel intra-uterine system; n.s. not significant

\section{Declarations}

\section{Ethics approval and consent to participate}

The study is approved by The Regional Committee on Health Research Ethics (H17017580) and the Data Protection Agency in the Capital Region of Denmark (2012-58-0004). All participants gave oral and written consent to participate in the study and were remunerated with 3,000 DKK after completing sample collection. All data were collected and managed using REDCap electronic data capture tools [38], hosted at the Capital Region of Denmark.

\section{Consent for publication}

Not applicable

\section{Availability of data and material}

The samples analysed in this study were submitted to the European Nucleotide

Archive under project number PRJEB37731, samples ERS4421369 - ERS4422941.

\section{Competing interests}

The authors report no competing interests.

\section{Funding}

This work was partly funded by Ferring Pharmaceuticals through a research collaboration with The Centre for Translational Microbiome Research (CTMR) at the Karolinska Institutet and HSN's research group at Rigshospitalet.

\section{Authors' contributions}

MCK, HSN - planned and organized study cohort, obtained ethics and data protection approval, collected samples, data management, wrote manuscript ZB - included participants and secured informed consent, collected samples, data management, wrote manuscript

ANA - collected samples, analysed data, wrote manuscript

LWH, EF, GE - analysed data, wrote the manuscript

ISK, LE - planned and organized the study cohort, wrote the manuscript

All authors have read and approved the final manuscript

\section{Acknowledgements (optional)}

We like to thank the nurses employed at the Recurrent Pregnancy Loss Unit in Copenhagen for their huge contribution with including participants: Louise Lunøe, Karen Kirchheiner and Marie Chonovitsch. We thank Marica Hamsten and Fredrik Boulund for invaluable contribution to sample handling logistics. 


\section{References}

[1] V. B. Young, "The role of the microbiome in human health and disease: An introduction for clinicians," BMJ (Online), vol. 356. BMJ Publishing Group, 2017, doi: 10.1136/bmj.j831.

[2] A. D. Kostic, R. J. Xavier, and D. Gevers, "The microbiome in inflammatory bowel disease: Current status and the future ahead," Gastroenterology, vol. 146, no. 6, pp. 1489-1499, 2014, doi: 10.1053/j.gastro.2014.02.009.

[3] G. Winter, R. A. Hart, R. P. G. Charlesworth, and C. F. Sharpley, "Gut microbiome and depression: What we know and what we need to know," Reviews in the Neurosciences, vol. 29, no. 6. Walter de Gruyter GmbH, pp. 629-643, Aug. 28, 2018, doi: 10.1515/revneuro-2017-0072.

[4] W. Hernández-Ceballos, J. Cordova-Gallardo, and N. MendezSanchez, "Gut Microbiota in Metabolic-associated Fatty Liver Disease and in Other Chronic Metabolic Diseases," J. Clin. Transl. Hepatol., vol. 000, no. 000, pp. 000-000, Mar. 2021, doi: 10.14218/jcth.2020.00131.

[5] A. S. Meijnikman, V. E. Gerdes, M. Nieuwdorp, and H. Herrema, "Evaluating causality of gut microbiota in obesity and diabetes in humans," Endocrine Reviews, vol. 39, no. 2. Oxford University Press, pp. 133-153, Apr. 01, 2018, doi: 10.1210/er.2017-00192.

[6] E. Figuero, Y. W. Han, and Y. Furuichi, "Periodontal diseases and adverse pregnancy outcomes: Mechanisms," Periodontology 2000, vol. 83, no. 1. Blackwell Munksgaard, pp. 175-188, Jun. 01, 2020, doi: 10.1111/prd.12295.

[7] K. A. Green, S. M. Zarek, and W. H. Catherino, "Gynecologic health and disease in relation to the microbiome of the female reproductive tract," Fertil. Steril., vol. 104, no. 6, pp. 13511357, 2015, doi: 10.1016/j.fertnstert.2015.10.010.

[8] S. J. Kroon, J. Ravel, and W. M. Huston, "Cervicovaginal microbiota, women's health, and reproductive outcomes," Fertil. Steril., vol. 110, no. 3, pp. 327-336, 2018, doi: 10.1016/j.fertnstert.2018.06.036.

[9] J. Ravel et al., "Vaginal microbiome of reproductive-age 
women," Proc. Natl. Acad. Sci. U. S. A., vol. 108, no. SUPPL. 1, pp. 4680-4687, Mar. 2011, doi: 10.1073/pnas.1002611107.

[10] T. Haahr, J. S. Jensen, L. Thomsen, L. Duus, K. Rygaard, and P. Humaidan, "Abnormal vaginal microbiota may be associated with poor reproductive outcomes: A prospective study in IVF patients," Hum. Reprod., vol. 31, no. 4, pp. 795-803, Apr. 2016, doi: 10.1093/humrep/dew026.

[11] R. Van Houdt, B. Ma, S. M. Bruisten, A. G. C. L. Speksnijder, J. Ravel, and H. J. C. De Vries, “Lactobacillus iners-dominated vaginal microbiota is associated with increased susceptibility to Chlamydia trachomatis infection in Dutch women: A casecontrol study," Sex. Transm. Infect., vol. 94, no. 2, pp. 117123, Mar. 2018, doi: 10.1136/sextrans-2017-053133.

[12] N. Brusselaers, S. Shrestha, J. van de Wijgert, and H. Verstraelen, "Vaginal dysbiosis and the risk of human papillomavirus and cervical cancer: systematic review and meta-analysis," American Journal of Obstetrics and Gynecology, vol. 221, no. 1. Mosby Inc., pp. 9-18.e8, Jul. 01, 2019, doi: 10.1016/j.ajog.2018.12.011.

[13] R. Romero et al., "The composition and stability of the vaginal microbiota of normal pregnant women is different from that of non-pregnant women," Microbiome, vol. 2, no. 1, Feb. 2014, doi: 10.1186/2049-2618-2-4.

[14] Y. Zhou et al., "Biogeography of the ecosystems of the healthy human body," Genome Biol., vol. 14, no. 1, Jan. 2013, doi: 10.1186/gb-2013-14-1-r1.

[15] C. Gosmann et al., "Lactobacillus-Deficient Cervicovaginal Bacterial Communities Are Associated with Increased HIV Acquisition in Young South African Women," Immunity, vol. 46 , no. 1, pp. 29-37, Jan. 2017, doi:

10.1016/j.immuni.2016.12.013.

[16] H. Borgdorff et al., "The association between ethnicity and vaginal microbiota composition in Amsterdam, the Netherlands," PLoS One, vol. 12, no. 7, p. e0181135, Jul. 2017, doi: 10.1371/journal.pone.0181135.

[17] V. L. Edwards et al., "The cervicovaginal microbiota-host interaction modulates chlamydia trachomatis infection," MBio, 
vol. 10, no. 4, 2019, doi: 10.1128/mBio.01548-19.

[18] J. Atashili, C. Poole, P. M. Ndumbe, A. A. Adimora, and J. S. Smith, "Bacterial vaginosis and HIV acquisition: A metaanalysis of published studies," AIDS, vol. 22, no. 12, pp. 14931501, Jul. 2008, doi: 10.1097/QAD.0b013e3283021a37.

[19] R. Koedooder et al., "The vaginal microbiome as a predictor for outcome of in vitro fertilization with or without intracytoplasmic sperm injection: A prospective study," Hum. Reprod., vol. 34, no. 6, pp. 1042-1054, Jun. 2019, doi: 10.1093/humrep/dez065.

[20] M. A. Elovitz et al., "Cervicovaginal microbiota and local immune response modulate the risk of spontaneous preterm delivery," Nat. Commun., vol. 10, no. 1, Dec. 2019, doi: 10.1038/s41467-019-09285-9.

[21] J. M. Fettweis et al., "The vaginal microbiome and preterm birth," Nat. Med., vol. 25, no. 6, pp. 1012-1021, 2019, doi: 10.1038/s41591-019-0450-2.

[22] M. Coudray and S. Kiplagat, "Bacterial vaginosis and the risk of human papillomavirus and cervical cancer," American Journal of Obstetrics and Gynecology, vol. 221, no. 2. Mosby Inc., pp. 171-172, Aug. 01, 2019, doi: 10.1016/j.ajog.2019.05.016.

[23] C. Balle et al., "Relationship between the oral and vaginal microbiota of South African adolescents with high prevalence of bacterial vaginosis," Microorganisms, vol. 8, no. 7, pp. 118, 2020, doi: 10.3390/microorganisms8071004.

[24] L. J. Forney, J. A. Foster, and W. Ledger, "The vaginal flora of healthy women is not always dominated by Lactobacillus species [3]," Journal of Infectious Diseases, vol. 194, no. 10. University of Chicago Press, pp. 1468-1469, Nov. 15, 2006, doi: 10.1086/508497.

[25] H. Kaur, M. Merchant, M. M. Haque, and S. S. Mande, “Crosstalk Between Female Gonadal Hormones and Vaginal Microbiota Across Various Phases of Women's Gynecological Lifecycle," Front. Microbiol., vol. 11, p. 551, Mar. 2020, doi: 10.3389/fmicb.2020.00551.

[26] R. J. Hickey et al., "Vaginal microbiota of adolescent girls prior 
to the onset of menarche resemble those of reproductive-age women," MBio, vol. 6, no. 2, Mar. 2015, doi: 10.1128/mBio.00097-15.

[27] A. L. Muhleisen and M. M. Herbst-Kralovetz, "Menopause and the vaginal microbiome," Maturitas, vol. 91, no. 2016, pp. 4250, 2016, doi: 10.1016/j.maturitas.2016.05.015.

[28] J. P. Brooks et al., "Effects of combined oral contraceptives, depot medroxyprogesterone acetate and the levonorgestrelreleasing intrauterine system on the vaginal microbiome," Contraception, vol. 95, no. 4, pp. 405-413, Apr. 2017, doi: 10.1016/j.contraception.2016.11.006.

[29] L. A. Vodstrcil et al., "Hormonal Contraception Is Associated with a Reduced Risk of Bacterial Vaginosis: A Systematic Review and Meta-Analysis," PLoS One, vol. 8, no. 9, 2013, doi: 10.1371/journal.pone.0073055.

[30] G. Donders, G. Bellen, D. Janssens, B. Van Bulck, P. Hinoul, and J. Verguts, "Influence of contraceptive choice on vaginal bacterial and fungal microflora," Eur. J. Clin. Microbiol. Infect. Dis., vol. 36, no. 1, pp. 43-48, Jan. 2017, doi: 10.1007/s10096016-2768-8.

[31] S. L. Achilles and S. L. Hillier, "The complexity of contraceptives: Understanding their impact on genital immune cells and vaginal microbiota," AIDS, vol. 27, no. SUIPPL.1, Oct. 2013, doi: 10.1097/QAD.0000000000000058.

[32] S. D. Song et al., "Daily Vaginal Microbiota Fluctuations Associated with Natural Hormonal Cycle, Contraceptives, Diet, and Exercise," mSphere, vol. 5, no. 4, pp. 1-14, 2020, doi: 10.1128/msphere.00593-20.

[33] G. G. G. Donders, G. Bellen, K. Ruban, and B. Van Bulck, "Short- and long-term influence of the levonorgestrelreleasing intrauterine system (Mirena ${ }^{\circledR}$ ) on vaginal microbiota and Candida," J. Med. Microbiol., vol. 67, no. 3, pp. 308-313, Mar. 2018, doi: 10.1099/jmm.0.000657.

[34] V. De Leo, M. C. Musacchio, V. Cappelli, P. Piomboni, and G. Morgante, "Hormonal contraceptives: Pharmacology tailored to women's health," Hum. Reprod. Update, vol. 22, no. 5, pp. 634-646, Sep. 2016, doi: 10.1093/humupd/dmw016. 
[35] G. Grandi, A. Farulla, F. G. Sileo, and F. Facchinetti, "Levonorgestrel-releasing intra-uterine systems as female contraceptives," Expert Opinion on Pharmacotherapy, vol. 19, no. 7. Taylor and Francis Ltd, pp. 677-686, May 03, 2018, doi: 10.1080/14656566.2018.1462337.

[36] N. Bostanci et al., "Dysbiosis of the Human Oral Microbiome During the Menstrual Cycle and Vulnerability to the External Exposures of Smoking and Dietary Sugar," Front. Cell. Infect. Microbiol., vol. 11, Mar. 2021, doi: 10.3389/fcimb.2021.625229.

[37] P. Gajer et al., "Temporal dynamics of the human vaginal microbiota," Sci. Transl. Med., vol. 4, no. 132, May 2012, doi: 10.1126/scitransImed.3003605.

[38] P. A. Harris et al., "The REDCap consortium: Building an international community of software platform partners," Journal of Biomedical Informatics, vol. 95. Academic Press Inc., Jul. 01, 2019, doi: 10.1016/j.jbi.2019.103208.

[39] D. E. Wood, J. Lu, and B. Langmead, “Improved metagenomic analysis with Kraken 2," Genome Biol., vol. 20, no. 1, Nov. 2019, doi: 10.1186/s13059-019-1891-0.

[40] J. Lu, F. P. Breitwieser, P. Thielen, and S. L. Salzberg, "Bracken: Estimating species abundance in metagenomics data," PeerJ Comput. Sci., vol. 2017, no. 1, p. e104, Jan. 2017, doi: 10.7717/peerj-cs.104.

[41] T. Chen, W. H. Yu, J. Izard, O. V. Baranova, A. Lakshmanan, and F. E. Dewhirst, "The Human Oral Microbiome Database: a web accessible resource for investigating oral microbe taxonomic and genomic information.," Database (Oxford)., vol. 2010, 2010, doi: 10.1093/database/baq013.

[42] L. W. Hugerth et al., "Assessment of In Vitro and In Silico Protocols for Sequence-Based Characterization of the Human Vaginal Microbiome," mSphere, vol. 5, no. 6, Dec. 2020, doi: 10.1128/msphere.00448-20.

[43] "CRAN - Package vegan." https://cran.r-project.org/web/packages/vegan/index.html (accessed Jun. 04, 2021).

[44] H. Mallick et al., "Multivariable Association Discovery in 
Population-scale Meta-omics Studies 3," bioRxiv, p. 2021.01.20.427420, Jan. 2021, doi:

10.1101/2021.01.20.427420.

[45] A. Hellström, K. Gemzell Danielsson, and H. Kopp Kallner, "Trends in use and attitudes towards contraception in Sweden: results of a nationwide survey," Eur. J. Contracept. Reprod. Heal. Care, vol. 24, no. 2, pp. 154-160, Mar. 2019, doi: 10.1080/13625187.2019.1581163.

[46] I. Lindh et al., "Contraceptive use in the Nordic countries," Acta Obstet. Gynecol. Scand., vol. 96, no. 1, pp. 19-28, Jan. 2017, doi: 10.1111/aogs.13055.

[47] "Products - Data Briefs - Number 327 - December 2018." https://www.cdc.gov/nchs/products/databriefs/db327.htm (accessed Jun. 03, 2021).

[48] E. K. Costello, C. L. Lauber, M. Hamady, N. Fierer, J. I. Gordon, and R. Knight, "Bacterial community variation in human body habitats across space and time," Science (80-. )., vol. 326, no. 5960, pp. 1694-1697, Dec. 2009, doi:

$10.1126 /$ science. 1177486 .

[49] N. Segata et al., "Composition of the adult digestive tract bacterial microbiome based on seven mouth surfaces, tonsils, throat and stool samples.," Genome Biol., vol. 13, no. 6, 2012, doi: 10.1186/gb-2012-13-6-r42.

[50] R. B. Jones et al., "Inter-niche and inter-individual variation in gut microbial community assessment using stool, rectal swab, and mucosal samples," Sci. Rep., vol. 8, no. 1, Dec. 2018, doi: 10.1038/s41598-018-22408-4.

[51] K. Fair et al., "Rectal Swabs from Critically III Patients Provide Discordant Representations of the Gut Microbiome Compared to Stool Samples," mSphere, vol. 4, no. 4, Jul. 2019, doi: 10.1128/msphere.00358-19.

[52] M. Fudaba, T. Kamiya, D. Tachibana, M. Koyama, and N. Ohtani, "Bioinformatics analysis of oral, vaginal, and rectal microbial profiles during pregnancy: A pilot study on the bacterial co-residence in pregnant women," Microorganisms, vol. 9, no. 5, 2021, doi: 10.3390/microorganisms9051027.

[53] M. A. D. Antonio, L. K. Rabe, and S. L. Hillier, "Colonization of 
the rectum by Lactobacillus species and decreased risk of bacterial vaginosis," J. Infect. Dis., vol. 192, no. 3, pp. 394398, Aug. 2005, doi: 10.1086/430926.

[54] H. A. Torky et al., "Relationship Between Ano-vaginal Distance and Bacterial Vaginosis (Cross-sectional Study)," Reprod. Sci., 2021, doi: 10.1007/s43032-021-00514-5.

[55] S. A. Roberts et al., "Mucosal lactoferrin response to genital tract infections is associated with iron and nutritional biomarkers in young Burkinabé women," Eur. J. Clin. Nutr., vol. 73 , no. 11 , pp. 1464-1472, 2019, doi: 10.1038/s41430019-0444-7.

[56] E. Amabebe and D. O. C. Anumba, "The vaginal microenvironment: The physiologic role of Lactobacilli," Frontiers in Medicine, vol. 5, no. JUN. Frontiers Media S.A., Jun. 01, 2018, doi: 10.3389/fmed.2018.00181.

[57] C. Heinemann and G. Reid, “Vaginal microbial diversity among postmenopausal women with and without hormone replacement therapy," Can. J. Microbiol., vol. 51, no. 9, pp. 777-781, Sep. 2005, doi: 10.1139/W05-070.

[58] P. D. Ginkel, D. E. Soper, R. G. Bump, and H. P. Dalton, "Vaginal Flora in Postmenopausal Women: The Effect of Estrogen Replacement," Infect. Dis. Obstet. Gynecol., vol. 1, no. 2, pp. 94-97, 1993, doi: 10.1155/S1064744993000225.

[59] G. J. Gerstner, W. Grünberger, E. Boschitsch, and M. Rotter, "Vaginal organisms in prepubertal children with and without vulvovaginitis - A vaginoscopic study," Arch. Gynecol., vol. 231, no. 3, pp. 247-252, Jun. 1982, doi: 10.1007/BF02110125.

[60] M. R. Hammerschlag et al., "Anaerobic microflora of the vagina in children.," Am. J. Obstet. Gynecol., vol. 131, no. 8, pp. 853-6, Aug. 1978, doi: 10.1016/s0002-9378(16)33130-1.

[61] G. W. McPherson et al., "The vaginal microbiome of transgender men," Clin. Chem., vol. 65, no. 1, pp. 199-207, Jan. 2019, doi: 10.1373/clinchem.2018.293654.

[62] D. A. Maclntyre et al., "The vaginal microbiome during pregnancy and the postpartum period in a European population," Sci. Rep., vol. 5, Mar. 2015, doi: 10.1038/srep08988. 
[63] L. Cheng et al., "Vaginal microbiota and human papillomavirus infection among young Swedish women," npj Biofilms Microbiomes, vol. 6, no. 1, pp. 1-10, 2020, doi: 10.1038/s41522-020-00146-8.

[64] L. Hardy, N. Cerca, V. Jespers, M. Vaneechoutte, and T. Crucitti, "Bacterial biofilms in the vagina," Res. Microbiol., vol. 168, no. 9-10, pp. 865-874, Nov. 2017, doi: 10.1016/j.resmic.2017.02.001.

[65] J. Castro, A. S. Rosca, P. Cools, M. Vaneechoutte, and N. Cerca, "Gardnerella vaginalis Enhances Atopobium vaginae Viability in an in vitro Model," Front. Cell. Infect. Microbiol., vol. 10, Mar. 2020, doi: 10.3389/fcimb.2020.00083.

[66] X. Chen, Y. Lu, T. Chen, and R. Li, "The Female Vaginal Microbiome in Health and Bacterial Vaginosis," Front. Cell. Infect. Microbiol., vol. 11, no. April, pp. 1-15, 2021, doi: 10.3389/fcimb.2021.631972. 